\title{
A generalized beta finite element method with coupled smoothing techniques for solid mechanics
}

\author{
W. Zeng ${ }^{\mathrm{a}, \mathrm{b}, *}$, G.R. Liu ${ }^{\mathrm{b}}$, C. Jiang ${ }^{\mathrm{b}, \mathrm{c}}$, T. Nguyen-Thoi ${ }^{\mathrm{d}}$, Y. Jiang ${ }^{\mathrm{e}}$ \\ ${ }^{\text {a } C E A S-B i o m e d i c a l ~ E n g i n e e r i n g ~(B M E), ~ U n i v e r s i t y ~ o f ~ C i n c i n n a t i, ~} 2901$ Woodside Dr., Cincinnati OH \\ 45221, USA \\ ${ }^{\mathrm{b}}$ CEAS-School of Aerospace Systems, University of Cincinnati, 2851 Woodside Dr., Cincinnati OH 45221, \\ $U S A$ \\ ' State Key Laboratory of Advanced Technology of Design and Manufacturing for Vehicle Body, Hunan \\ University, China, 410082 \\ ${ }^{\mathrm{d}}$ Division of Computational Mathematics and Engineering (CME), Institute for Computational Science \\ (INCOS), Ton Duc Thang University, Hochiminh City, Vietnam \\ ${ }^{\mathrm{e}}$ Hefei General Machinery Research Institute, 888 West Changjiang Rd., Hefei 230031, China
}

\begin{abstract}
This paper presents a generalized smoothing techniques based beta finite element method $(\beta$ FEM) to improve the performance of standard FEM and the existing smoothed finite element methods (S-FEM) in solid mechanics. As we know, the edge-based (for 2D) or face-based (for 3D) strain smoothing techniques can bring much more accurate solutions than standard FEM, and offer lower bounds for force driven problems. The node-based smoothing technique with "overly-soft" feature, on the other hand has a unique property of producing upper bound solutions. This work proposes a novel generalized S-FEM with the smoothing domains generated based on both edges/faces and nodes. An adjustable parameter $\beta$ is introduced to control the ratio of the area of edge/face-based and node-based smoothing domains. It is found that nearly exact
\end{abstract}

\footnotetext{
*Correspondence to: W. Zeng, CEAS-Biomedical Engineering (BME), University of Cincinnati, 2901 Woodside Dr., Cincinnati OH 45221, USA.

E-mail: zengwe@mail.uc.edu, zeng.work@gmail.com
}

(C) 2016. This manuscript version is made available under the Elsevier user license http://www.elsevier.com/open-access/userlicense/1.0/ 
solutions in strain energy can be obtained by tuning the parameter, making use of the important property that the exact solution is bonded by the solutions of NS-FEM and ES/FS-FEM. Standard patch tests are likewise satisfied. A number of numerical examples (static, dynamic, linear and nonlinear) have shown that the present $\beta$ FEM method is found to be ultra-accurate, insensitive to mesh quality, temporal stable, capable of modeling complex geometry, immune from volumetric locking, etc.

Keywords: Computational methods; Strain smoothing techniques; Generalized beta finite element method ( $\beta$ FEM); Vibration analysis; Large deformation; Solution bound.

\section{Introduction}

The standard constant finite elements such as 3-node triangular or 4-node tetrahedral elements (T-elements) were popular and preferred in practical mechanics problems for many years, as they offer many advantages such as convenience in FE implementation, high mesh quality, adaptive analysis with mesh rezoning, etc. And sometimes triangular/ tetrahedral mesh (T-mesh) may be the only option for mesh generation of complex geometries (e.g., biomechanical problems with irregular geometrical shapes). However, compared with quadrilateral/hexahedral meshes, the T-mesh using constant strain T-elements has its own numerical drawbacks including the inaccuracy, shear and volumetric locking due to excessive stiffness, especially for large deformation problems. As such, it is usually not recommended to use T-mesh in commercial FEM software packages.

In order to overcome the volumetric locking for plane strain problems and poor accuracy in stress solution, some new FEM approaches have been developed including supplementing the element displacement field with additional nodes and utilizing reduced numerical integration rules to calculate the element stiffness matrix. However, these procedures are not applicable or compatible with constant strain T-elements. T-mesh with 
second-order or higher-order elements is thought to a good option to avoid the locking issues, but it may be ineffective for extremely large deformation problems due to the intermediate nodes $[1,2]$. In order to dealing with these element defects of T-mesh, a number of researchers made their efforts to improve it in the past 30 years. For example, Allman $[3,4]$ improved the accuracy of triangle elements by using vertex connectors which included rotations. However, it exhibited an unusual type of zero energy mode, in addition to the rigid body movements. Reference [5] made a critical assessment of the Allman's triangular membrane element with drilling degrees of freedom via examining the performance of the element combined with a triangular plate bending element. Huang et al. [6] modified Allman's triangular planar element with drilling degree of freedom and dealt with spurious energy mode by an introduced constraint which ensures that the drilling degree of freedom is a true rotation in elasticity. Piltner and Taylor [7] developed the enhanced triangle elements to deal with nearly incompressible plane strain problems. However, the requirement of more degrees of freedom has limited the practical applications of these methods. In References [8,9], elements with rotational freedom were also designed to improve the bending performance or stiffness matrices for planar triangular elements. Reference [10] proposed a weighted least-squares formulation for deriving constant strain T-elements, which claimed to be possible to eliminate volumetric locking. In Reference [11], it introduced a node-based uniform strain element for T-mesh and it is capable in avoiding the volumetric locking and reducing the effects of shear locking for static linear elastic problems. Reference [12] used bubble function displacements in conjunction with the assumed strain formulation to construct triangular solid shell elements for precluding membrane locking effect.

In the past several years, Liu and his group developed smoothed finite element methods (S-FEM) [13-19] by introducing the gradient/strain smoothing techniques to FEM settings and using direct (no mapping) point interpolation for computing shape functions. The gradient/strain smoothing techniques using Green's theorem have been 
exploited in the past few decades for the quasi-conforming elements for plates and shells [20], stabilizing nodal integration of meshfree methods [21,22] and natural element method [23]. The essential idea of S-FEM is to utilize a standard first-order finite element mesh (in particular T-mesh) to build numerical models with good performance [24]. In S-FEM, the compatible strain field is constructed in a Galerkin weak form model to produce some good properties. Compared with the element-based implementation in the standard FEM, the S-FEM models evaluate the weak form based on smoothing domains. The smoothing domains can be constructed within the elements but usually beyond the elements, which is able to bring in the information of the neighboring elements. According to different fashions in the creation of smoothing domains, several different types of S-FEM models have been proposed: the cell-based smoothed FEM (CS-FEM) [16,19], node-based smoothed FEM (NS-FEM) [18], edge based smoothed FEM (ES-FEM) [15,17], face-based smoothed FEM (FS-FEM) [25], etc. Compared with the standard FEM, the overestimation behavior of stiffness values shall be reduced or alleviated in S-FEM and it significantly improves the accuracy of both primal and dual quantities [26]. In addition, the evaluation of shape function derivatives involved in FEM is avoided in S-FEM. The applications of S-FEM models in elasticity have shown they are insensitive to mesh distortion (compared with standard FEM) due to the absence of isoparametric mapping [27,28]. Furthermore, an S-FEM model can use the same background mesh as the standard FEM model, which does not require introducing additional degrees of freedom.

The intensive numerical studies have already demonstrated that the class of S-FEM models shows some advantages over standard FEM [24]. Among these S-FEM models, the ES-FEM (or FS-FEM for 3D) possesses some properties such as: i) ES/FS-FEM can produce solution with properties of super-convergence and higher accuracy compared with corresponding FEM model; ii) it usually generates lower bound to the exact solution in text of strain energy, but still has the feature of overestimation of stiffness; iii) it can 
use T-mesh which can be conveniently generated especially for complex geometries; iv) the ES/FS-FEM models are always stiffer than NS-FEM or FEM, partially due to the number of edges is always larger than the number of nodes with the same background mesh; v) the vibration models using ES/FS-FEM are often temporally stable and there are no spurious non-zeros energy modes found in free vibration analysis [24]. Meanwhile, the NS-FEM has some interesting properties [29-31]: i) it has the unique upper bound property in strain energy as it may extremely soften the over-stiffness of the corresponding standard FEM model; ii) it achieves accurate and often super-convergent stress solutions; iii) it is effective in overcoming volumetric locking; iv) it works effectively with T-mesh; v) it performs spatially stable but may be temporally instable with non-zero-energy spurious modes.

Considering the fact that the ES-FEM is capable of producing the accurate solution from the lower bound (better than standard FEM) and the NS-FEM can approximate the solution from the upper bound, a generalized mixed smoothed FEM model can be naturally conceived in order to obtain the exact or close-to-exact solution measured in an energy norm. Another fascinating aspect is that the generalized smoothed FEM can be versatile and may inherit the merits from both the NS-FEM and ES/FS-FEM. In this work, a novel ultra-accurate generalized smoothing techniques based beta finite element method $(\beta \mathrm{FEM})$ based on T-mesh is developed and then applied in different mechanics problems, particularly for 3D solid mechanics problems. In $\beta \mathrm{FEM}$, the smoothing domains will be constructed by a mixed fashion of node-based and edge/face-based smoothing techniques, in which the adjustable parameter $\beta \in[0,1]$ tunes the portion of area of the node-based and edge-based smoothing domains. The idea of $\beta$ FEM can be regarded as a utilization of the overestimation property of ES/FS-FEM and the unique under-estimation property of NS-FEM using T-elements, and hence it can be "tuned" to have good features of both methods. Since both the NS-FEM and ES/FS-FEM with T-elements are spatially stable [24], the presented $\beta$ FEM will be spatial stable and the convergence can be guaranteed. In 
addition, the scheme ensures the variational consistence and the compatibility of the displacement field, which ensures reproducing linear field exactly [32-34].

The paper aims to propose and formulate the novel generalized $\beta$ FEM for solid mechanics problems with first-order triangular/tetrahedral mesh, using the mixed edge /face-based and node-based strain smoothing techniques. The governing equation and different smoothing techniques utilized in this work will be briefly introduced in Section 2. The idea of $\beta$ FEM for both $2 \mathrm{D}$ and 3D problems will be presented in Section 3. Section 4 considers the implementation aspects for vibration analysis and large deformation problems which will be shown in subsequent numerical examples. The standard patch test and numerical examples will be discussed in Section 5 and 6.The conclusion will be summarized in the last section.

\section{Background of the problem and strain smoothing techniques}

The target of our method is to solve the solid mechanics problems using the weakened weak $\left(\mathrm{W}^{2}\right)$ Galerkin formulation [33]. For example, we can consider an elastic deformable body occupying domain $\Omega$, subjected to the body force $\mathbf{f}^{b}$ and the traction $\mathbf{f}^{t}$ on the natural boundary $\Gamma_{t}$. The object undergoes arbitrary virtual displacements with the compatible virtual strains $\delta \boldsymbol{\varepsilon}$ and internal displacement $\delta \mathbf{u}$. The dynamic equilibrium equations, which contain the inertial and damping forces, can be described in the following form:

$$
\int_{\Omega} \delta \boldsymbol{\varepsilon}^{\mathrm{T}} \mathbf{D} \boldsymbol{\varepsilon} \mathrm{d} \Omega-\int_{\Omega} \delta \mathbf{u}^{\mathrm{T}}[\mathbf{b}-\rho \ddot{\mathbf{u}}-c \dot{\mathbf{u}}] \mathrm{d} \Omega-\left(\int_{\Omega} \delta \mathbf{u}^{\mathrm{T}} \mathbf{f}^{b} \mathrm{~d} \Omega+\int_{\Gamma_{t}} \delta \mathbf{u}^{\mathrm{T}} \mathbf{f}^{t} \mathrm{~d} \Gamma\right)=0
$$

where $\mathbf{D}$ is the Hooke matrix of elastic constants which is related to modulus $E$ and Poisson's ratio $v$. For static problem, the second term in Eq. (1) will be vanished. The strain tensor $\boldsymbol{\varepsilon}$ can be expressed by displacement $\mathbf{u}$ using compatibility relation: 


$$
\boldsymbol{\varepsilon}=\nabla_{S} \mathbf{u}(\mathbf{x})
$$

where $\nabla_{S}$ is the symmetric gradient of the displacement field. In FEM, the displacement $\mathbf{u}$ and arbitrary virtual displacements $\delta \mathbf{u}$ will be approximated using trial functions by following expression:

$$
\mathbf{u}^{h}(\mathbf{x})=\sum_{I=1}^{\mathrm{NP}} \mathbf{N}_{I}(x) \mathbf{d}_{I} \quad \text { and } \delta \mathbf{u}^{h}(\mathbf{x})=\sum_{I=1}^{\mathrm{NP}} \mathbf{N}_{I}(x) \delta \mathbf{d}_{I}
$$

where $\mathbf{N}_{I}$ represents the a matrix of shape functions, $\mathbf{d}_{I}$ is the vector of the associated nodal displacements, and NP is the total number of the nodal variables of the element.

The S-FEM techniques based FEM models evaluate the smoothed strain based on associated smoothing domains and the manipulation can be derived from the compatible strains of finite element expressed in Eq. (2). Hereafter in this section, the smoothing operation and associated fashions of strain smoothing techniques are presented.

\subsection{Local gradient smoothing operation}

The strain smoothing technique, which was firstly employed in the Galerkin meshfree methods, was conceived from the moving least-squares (MLS) and reproducing kernel approximations [21,35]. The strain smoothing operation is manipulated over the local smoothing domain created within elements (e.g., CS-FEM) or beyond the elements (e.g., NS-FEM, ES-FEM and FS-FEM). Generally, the smoothed strain field $\tilde{\boldsymbol{\varepsilon}}_{k}$ will be calculated from a weighted average of the standard FEM strain field $\varepsilon^{h}(\mathbf{x})$. If a problem domain is discretized into some representative smoothing domains $\Omega_{k}^{s}(k=1,2, \ldots)$ with their boundaries $\Gamma_{k}^{s}(k=1,2, \ldots)$, it requires these domains be non-overlapping and non-gap. To smooth the strain field (the gradient of the displacement field) at a point in a smoothed domain $\Omega_{k}^{s}$, it can be computed via 


$$
\tilde{\boldsymbol{\varepsilon}}_{k}\left(\mathbf{x}_{C}\right)=\int_{\Omega^{h}} \boldsymbol{\varepsilon}^{h}(\mathbf{x}) \Phi_{k}\left(\mathbf{x}-\mathbf{x}_{C}\right) \mathrm{d} \Omega
$$

where $\Phi_{k}\left(\mathbf{x}-\mathbf{x}_{C}\right)$ is a smoothing function or a distribution function which satisfies the unity property:

$$
\Phi_{k}\left(\mathbf{x}-\mathbf{x}_{C}\right) \geq 0 \text { and } \int_{\Omega_{k}^{s}} \Phi_{k}\left(\mathbf{x}-\mathbf{x}_{C}\right) \mathrm{d} \Omega=1
$$

We can utilize the Heaviside-type piecewise constant smoothing function expressed as follows [24]:

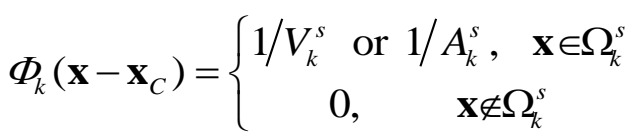

where $V_{k}^{s}=\int_{\Omega_{k}^{s}} \mathrm{~d} \Omega$ (or $A_{k}^{s}=\int_{\Omega_{k}^{s}} \mathrm{~d} \Omega$ for $2 \mathrm{D}$ smoothing domains) is the volume (or area) of the smoothing domain $\Omega_{k}^{s}$. Substituting Eq. (6) into Eq. (4) and introducing the divergence theorem, the smoothed strains have the form

$$
\tilde{\boldsymbol{\varepsilon}}_{k}=\frac{1}{V_{k}^{s}} \int_{\Omega_{k}^{s}} \nabla_{S} \mathbf{u}^{h} \mathrm{~d} \Omega=\frac{1}{V_{k}^{s}} \int_{\Gamma_{k}^{s}} \mathbf{n}_{k}^{s}(\mathbf{x}) \mathbf{u}^{h}(\mathbf{x}) \mathrm{d} \Gamma
$$

where $V_{k}^{s}$ shall be replaced by $A_{k}^{s}$ for $2 \mathrm{D}$ smoothing domains, $\Gamma_{k}^{s}$ is the boundary of the smoothing domain $\Omega_{k}^{s}$, and $\mathbf{n}_{k}^{s}(\mathbf{x})$ denotes the outward normal matrix on the boundary $\Gamma_{k}^{s}$ given as

$$
\mathbf{n}_{k}^{s}(\mathbf{x})=\left[\begin{array}{cc}
n_{k x}^{s} & 0 \\
0 & n_{k y}^{s} \\
n_{k y}^{s} & n_{k x}^{s}
\end{array}\right] \text { for 2D, or } \mathbf{n}_{k}^{s}(\mathbf{x})=\left[\begin{array}{ccc}
n_{k x}^{s} & 0 & 0 \\
0 & n_{k y}^{s} & 0 \\
0 & 0 & n_{k z}^{s} \\
n_{k y}^{s} & n_{k x}^{s} & 0 \\
0 & n_{k z}^{s} & n_{k y}^{s} \\
n_{k z}^{s} & 0 & n_{k x}^{s}
\end{array}\right] \text { for 3D }
$$

where $n_{k x}^{s}, n_{k y}^{s}$ and $n_{k z}^{s}$ are the unit outward normal components along the $x$-axis, $y$-axis and $z$-axis, respectively. 


\subsection{Briefing of edge-based strain smoothing}

In the ES-FEM, the smoothing domains are constructed in association with the edges of the three-node triangle elements as illustrated in Fig. 1. These smoothing domains are generated based upon the edges of the elements such that $\Omega \approx \bigcup_{k=1}^{N_{e d}} \Omega_{k}^{s}$ and $\Omega_{i}^{s} \cap \Omega_{j}^{s}=\varnothing$ for $i \neq j$, in which $N_{e d}$ denotes the number of edges for all elements.

For triangular elements, the smoothing domain $\Omega_{k}^{s}$ around the edge $k$ ( $k=l$ for boundary edges and $k=m$ for interior edges) is constructed by connecting the centroid(s) of corresponding adjacent element(s) and the endpoints of the edge as shown in Fig. 1.

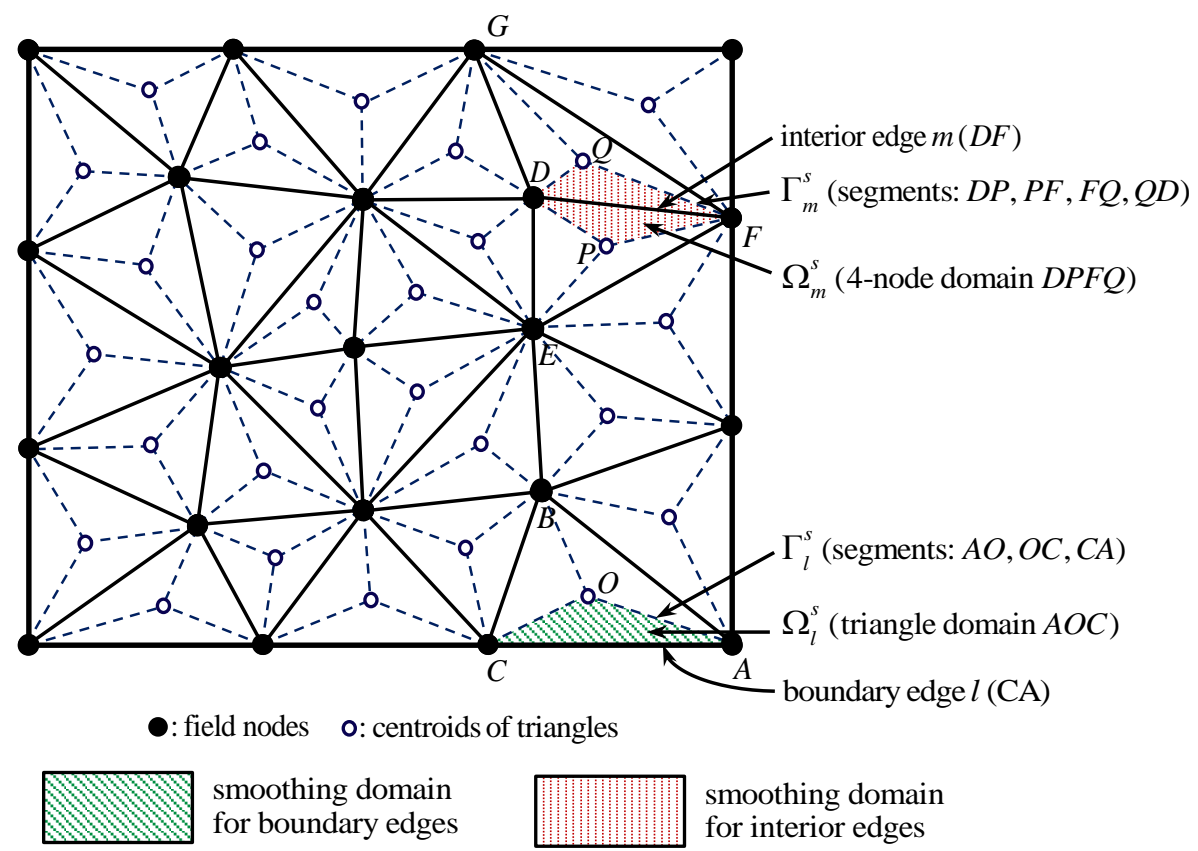

Fig. 1. The edge-based smoothing domains constructed upon triangular elements. For a boundary edge $l$, the smoothing domain $\Omega_{l}^{s}$ is a triangle $A O C$. While for an interior edge $m$, the smoothing domain $\Omega_{m}^{s}$ is the four-sided convex polygon $D P F Q$. 
If we create a background mesh using triangular elements, the following matrix form of the smoothed strain term in Eq.(7) for $\Omega_{k}^{s}$ can be formulated:

$$
\tilde{\boldsymbol{\varepsilon}}_{k}=\sum_{I \in S_{k}^{S}} \tilde{\mathbf{B}}_{I} \mathbf{d}_{I}
$$

where $S_{k}^{s}$ is the set of supporting nodes for $\Omega_{k}^{s}$, i.e., the set of all nodes for the elements comprised the common edge $k$. For the representative ES-FEM smoothing domains as shown in Fig. 1, $S_{k}^{s}$ stands for the set of nodes $\{A, B, C\}$ associated with the boundary edge $A C$, and $\{D, E, F, G\}$ stands for the interior edge $D F . \tilde{\mathbf{B}}_{I}$ denotes the smoothed strain-displacement matrix which has the form:

$$
\tilde{\mathbf{B}}_{I}=\frac{1}{A_{k}^{s}} \int_{\Gamma_{k}^{s}} \mathbf{n}_{k}^{s}(\mathbf{x}) \mathbf{N}_{I}(\mathbf{x}) \mathrm{d} \Gamma=\left[\begin{array}{ccc}
\tilde{b}_{I x} & 0 & \tilde{b}_{I y} \\
0 & \tilde{b}_{I y} & \tilde{b}_{I x}
\end{array}\right]^{T}
$$

with

$$
\tilde{b}_{I h}=\frac{1}{A_{k}^{s}} \int_{\Gamma_{k}^{s}} N_{I}(\mathbf{x}) n_{k h}^{s}(\mathbf{x}) \mathrm{d} \Gamma, h=x, y
$$

We can find that only the shape functions $N_{I}$, without their derivatives, are required for the computation in the above equation. For a linearly compatible displacement field used along $\Gamma_{k}^{s}$, the numerical integration along each boundary segment $\Gamma_{k, t}^{s}$ may only require a single Gaussian point. Based upon the Gauss quadrature, we can use the following expression

$$
\tilde{b}_{I h}=\frac{1}{A_{k}^{s}} \sum_{t=1}^{n_{\Gamma}^{s}} N_{I}\left(\mathbf{x}_{t}^{G P}\right) n_{k h, t}^{s} l_{k, t}^{s}, \quad h=x, y
$$

where $n_{\Gamma}^{s}$ denotes the total number of boundary segments $\Gamma_{k, t}^{s} \subset \Gamma_{k}^{s}$, In Fig. 1, the smoothing domain associated with the boundary edge $l(A C)$ includes three boundary segments $(A O, O C, C A)$, i.e., $n_{\Gamma}^{s}=3$; and it includes four segments $(D P, P F, F Q, Q D)$ 
for the interior edge $m(D F)$, i.e., $n_{\Gamma}^{s}=4 . \mathbf{x}_{t}^{G P}$ denotes the midpoint or Gaussian point for the boundary segment $\Gamma_{k, t}^{s}, l_{k, t}^{s}$ and $n_{k h, t}^{s}$ represent the length and outward unit normal of $\Gamma_{k, t}^{s}$, respectively. The area of smoothing domain in these equations, $A_{k}^{s}$, will be calculated by:

$$
A_{k}^{s}=\int_{\Omega_{k}^{s}} d \Omega=\frac{1}{3} \sum_{j=1}^{n_{k}^{e}} A_{j}^{e}
$$

where the number of elements, $n_{k}^{e}$, attached to the edge $k$ can be $n_{k}^{e}=1$ for the boundary edges, or can be $n_{k}^{e}=2$ for interior edges (Fig. 1).

Based on the expression of smoothed strain field in Eq. (7), the smoothed strain-displacement matrix in Eq. (10) can be rewritten by the average of strain-displacement matrix $\mathbf{B}_{I}(\mathbf{x})$ of the involved element(s):

$$
\tilde{\mathbf{B}}_{I}=\frac{1}{A_{k}^{s}} \int_{\Gamma_{k}^{s}} \mathbf{n}_{k}^{s}(\mathbf{x}) \mathbf{N}_{I}(\mathbf{x}) \mathrm{d} \Gamma=\frac{1}{A_{k}^{s}} \int_{\Omega_{k}^{s}} \nabla_{S} \mathbf{N}_{I}(\mathbf{x}) \mathrm{d} \Omega=\frac{1}{A_{k}^{s}} \int_{\Omega_{k}^{s}} \mathbf{B}_{I}(\mathbf{x}) \mathrm{d} \Omega
$$

where the line integral along $\Gamma_{k}^{s}$ is converted to area integration in $\Omega_{k}^{s}$ based on the divergence theorem. The smoothed strain-displacement matrix for ES-FEM can be computed by

$$
\tilde{\mathbf{B}}_{I}=\frac{1}{A_{k}^{s}} \sum_{j=1}^{n_{k}^{e}} \frac{1}{3} A_{j}^{e} \mathbf{B}_{j}^{e}
$$

where $\mathbf{B}_{j}^{e}=\sum_{I \in S_{j}^{e}} \mathbf{B}_{I} \quad$ stands for the compatible strain-displacement matrix for $j^{\text {th }}$ element associated with the edge $k$. The strain-displacement matrix $\mathbf{B}_{I}(\mathbf{x})$ for the node $I$ in a triangle element can be evaluated as 


$$
\mathbf{B}_{I}(\mathbf{x})=\nabla_{S} \mathbf{N}_{I}(\mathbf{x})=\left[\begin{array}{ccc}
\frac{\partial N_{I}(\mathbf{x})}{\partial x} & 0 & \frac{\partial N_{I}(\mathbf{x})}{\partial y} \\
0 & \frac{\partial N_{I}(\mathbf{x})}{\partial y} & \frac{\partial N_{I}(\mathbf{x})}{\partial x}
\end{array}\right]^{T}
$$

Since the background triangular elements employ linear shape functions, the entries of matrix $\mathbf{B}_{I}(\mathbf{x})$ will be constants, and so are the entries for the matrix $\mathbf{B}_{j}^{e}$ and $\tilde{\mathbf{B}}_{I}$.

\subsection{Briefing of face-based strain smoothing}

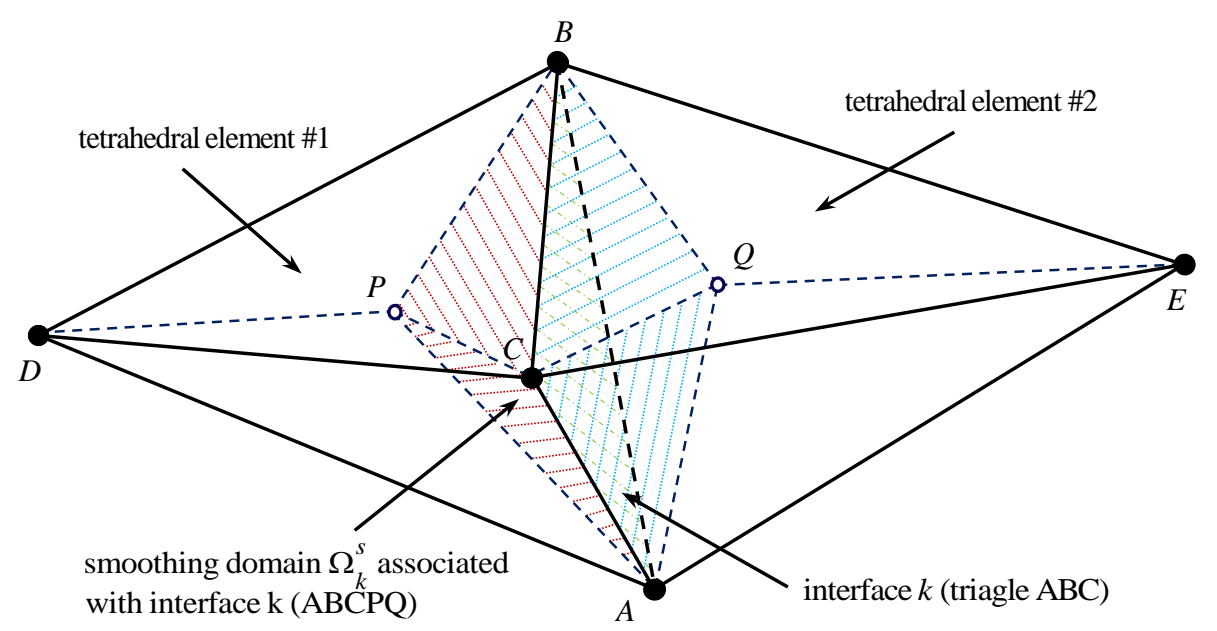

Fig. 2. A face-based smoothing domain $\Omega_{k}^{s}$ constructed from two adjacent tetrahedral elements based on their interface $k$.

Analogous to the 2D ES-FEM, the FS-FEM creates smoothing domains associated with faces of tetrahedral elements such that $\Omega \approx \bigcup_{k=1}^{N_{\text {fuce }}} \Omega_{k}^{s}$ and $\Omega_{i}^{s} \cap \Omega_{j}^{s}=\varnothing$ for $i \neq j$, in which $N_{\text {face }}$ is the number of faces for all elements in the problem domain. For tetrahedral elements sketched in Fig. 2, the smoothing domain associated with the face $k$ can be constructed through connecting the triangle vertices $(A, B$ and $C$ ) and the two centroids of the two attached elements ( $P$ and $Q$ ), i.e., the triangular bipyramid. By 
applying the face-based smoothing operation [36], the smoothed strain-displacement matrix may have a similar formulation expressed as

$$
\tilde{\mathbf{B}}_{I}=\frac{1}{V_{k}^{s}} \sum_{j=1}^{n_{k}^{e}} \frac{1}{4} V_{j}^{e} \mathbf{B}_{j}^{e}
$$

where $n_{k}^{e}$ is the number of elements attached to the face $\left(n_{k}^{e}=1\right.$ for the boundary faces and $n_{k}^{e}=2$ for interior faces), $\mathbf{B}_{j}^{e}$ is the standard compatible strain-displacement matrix for $j^{\text {th }}$ element attached to face $k$. The matrix $\mathbf{B}_{I}(\mathbf{x})$ for the node $I$ in tetrahedral elements is expressed as

$$
\mathbf{B}_{I}(\mathbf{x})=\nabla_{S} \mathbf{N}_{I}(\mathbf{x})=\left[\begin{array}{cccccc}
\frac{\partial N_{I}(\mathbf{x})}{\partial x} & 0 & 0 & \frac{\partial N_{I}(\mathbf{x})}{\partial y} & 0 & \frac{\partial N_{I}(\mathbf{x})}{\partial z} \\
0 & \frac{\partial N_{I}(\mathbf{x})}{\partial y} & 0 & \frac{\partial N_{I}(\mathbf{x})}{\partial x} & \frac{\partial N_{I}(\mathbf{x})}{\partial z} & 0 \\
0 & 0 & \frac{\partial N_{I}(\mathbf{x})}{\partial z} & 0 & \frac{\partial N_{I}(\mathbf{x})}{\partial y} & \frac{\partial N_{I}(\mathbf{x})}{\partial x}
\end{array}\right]^{T}
$$

\subsection{Briefing of node-based strain smoothing}

The node-based strain smoothing technique constructs smoothing domains for the strain field associated with nodes [37], which is different from edge-based strain smoothing technique. Here we only introduce 3D node-based strain smoothing technique, which is seldom found in existing references. In Fig. 3, the node-based smoothing domain $\Omega_{q}^{s}$ in element $j$ (sub-smoothing domain $\Omega_{q, j}^{s}$ ) is created by connecting the centroid of the tetrahedral element $j$, centroids of the three associated faces $(\triangle A B C$, $\triangle A B D$ and $\triangle A C D$ ) and the middle points of associated edges, i.e., polyhedron AELGMFKO. The set of supporting nodes $S_{q}^{s}$ of node-based smoothing domain includes all the nodes belonging to the surrounding elements for node $q$, e.g., the field nodes $\{A, B, C, D\}$ for the sub-smoothing domain $\Omega_{q, j}^{s}$ in Fig. 3 and other associated 
nodes for those elements which are not shown in Fig. 3 but share the node $q$. For the domain, it is viewed as the combination of the sub-domains of all these elements connected to the node $q$. The smoothed strain-displacement matrix $\overline{\mathbf{B}}_{I}$ of NS-FEM can be computed by a similar fashion of smoothing operation as the ES-FEM (Eq. (10)(14)), specified by

$$
\overline{\mathbf{B}}_{I}=\frac{1}{V_{q}^{s}} \sum_{l=1}^{n_{q}^{e}} \frac{1}{4} V_{l}^{e} \mathbf{B}_{l}^{e} \quad \text { with } \quad V_{q}^{s}=\int_{\Omega_{q}^{s}} d \Omega=\frac{1}{4} \sum_{l=1}^{n_{q}^{e}} V_{l}^{e}
$$

where $n_{q}^{e}$ represents the number of elements associated with node $q, V_{l}^{e}$ denotes the area of the $l^{\text {th }}$ element associated with the node $q$. The compatible strain-displacement matrix $\mathbf{B}_{l}^{e}$ for the $l^{\text {th }}$ element around the node $q$ has the form $\mathbf{B}_{l}^{e}=\sum_{I \in S_{l}^{e}} \mathbf{B}_{I}$. And the $\mathbf{B}_{I}$ for node $I \in S_{q}^{s}$ is defined by the form of Eq. (18) for tetrahedral background elements. 


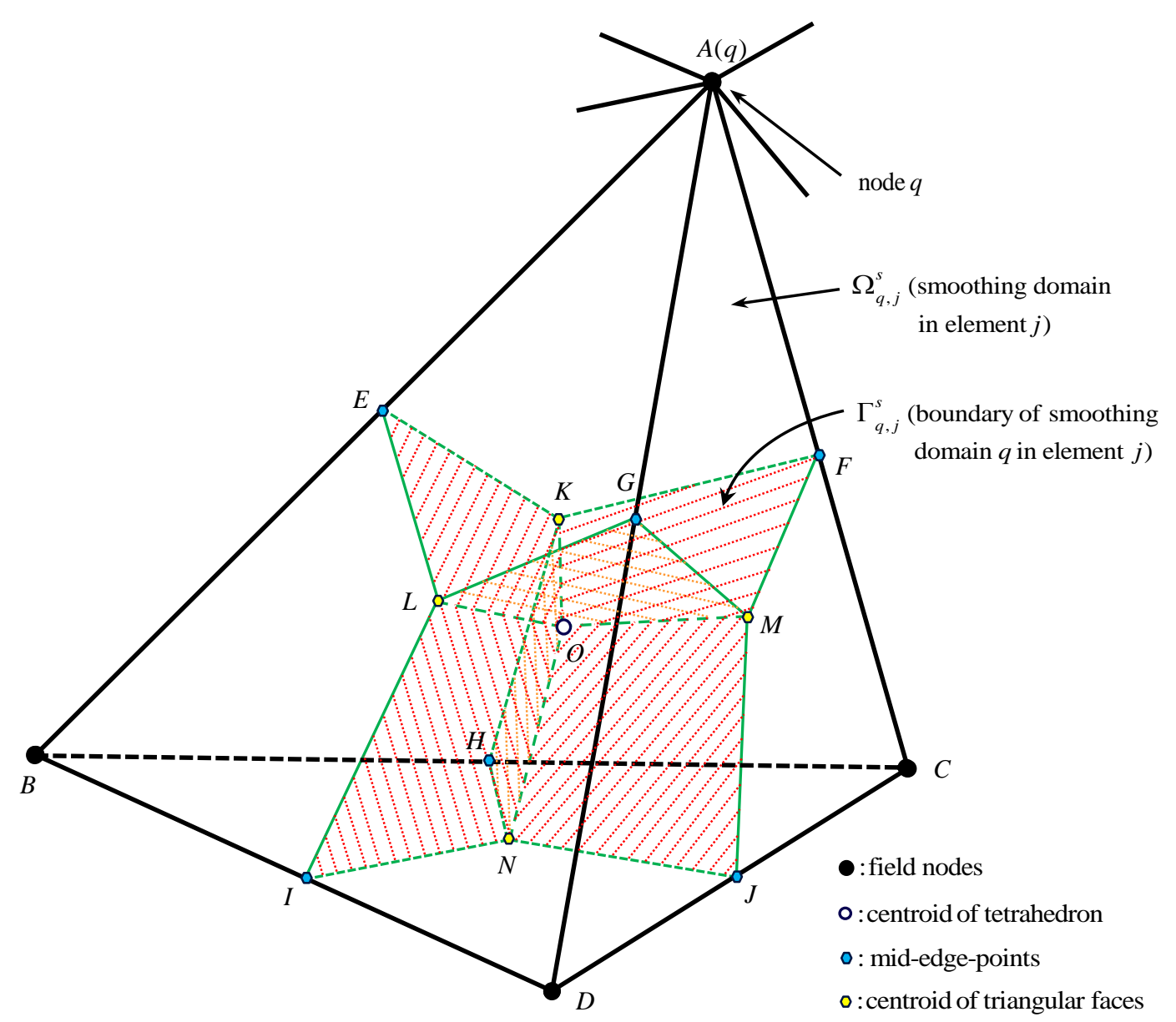

Fig. 3. Division of tetrahedral elements into 3D node-based smoothing domains. For example, the sub-smoothing domain $\Omega_{q, j}^{s}$ for node $q$ in the element $j$ is a polyhedron AELGMFKO.

For 2D NS-FEM, the implementation is quite similar as the 3D NS-FEM and the variable of volume needs to be replaced by associated area in formulation in Eq. (19). The set of supporting nodes $S_{q}^{s}$ will be all of the nodes belong to the elements which contain this node $q$.

\section{The idea of generalized beta finite element method}




\subsection{The idea of $\beta F E M$}

In order to describe the implementation of $\beta \mathrm{FEM}$ using T-mesh, here we choose 3D tetrahedral background elements as our example. The background elements (shown in Fig. 4) are further divided into two different types of smoothing domains: the node-base smoothing domains (the domain surrounding a node, e.g., node $A$ as shown in Fig. 4) and the face-based smoothing domains (the domains attached a face). The portion of volume of face-based and node-based smoothing domains will be tuned by a parameter $\beta$. In Fig. 4, the length of an edge of a triangle/tetrahedral element (e.g., $A C$ ) is assumed to be " $L$ ". If we adopt the scale factor $\beta$ to tune the dividing points ( $P$ and $Q$ ), the length of segments on the edge has the relations such as: $l_{1}=l_{3}=\frac{1}{2} \beta L$ and $l_{2}=(1-\beta) L$. If we take a 3D problem as our example, the volume of the four sub-domains by node-based smoothing technique in an element (four hexahedrons at four corners of a tetrahedral element $k$ ) will be $V_{q, j}^{s}=\frac{1}{4} \beta^{3} V_{k}^{e}$. Then a face-based sub-smoothing domain attached to the face $k$ in the element, i.e., domains attached to the " $Y$ " shape on the face $k$ in Fig. 4, has the volume $\frac{1}{4}\left(1-\beta^{3}\right) V_{k}^{e}$. 


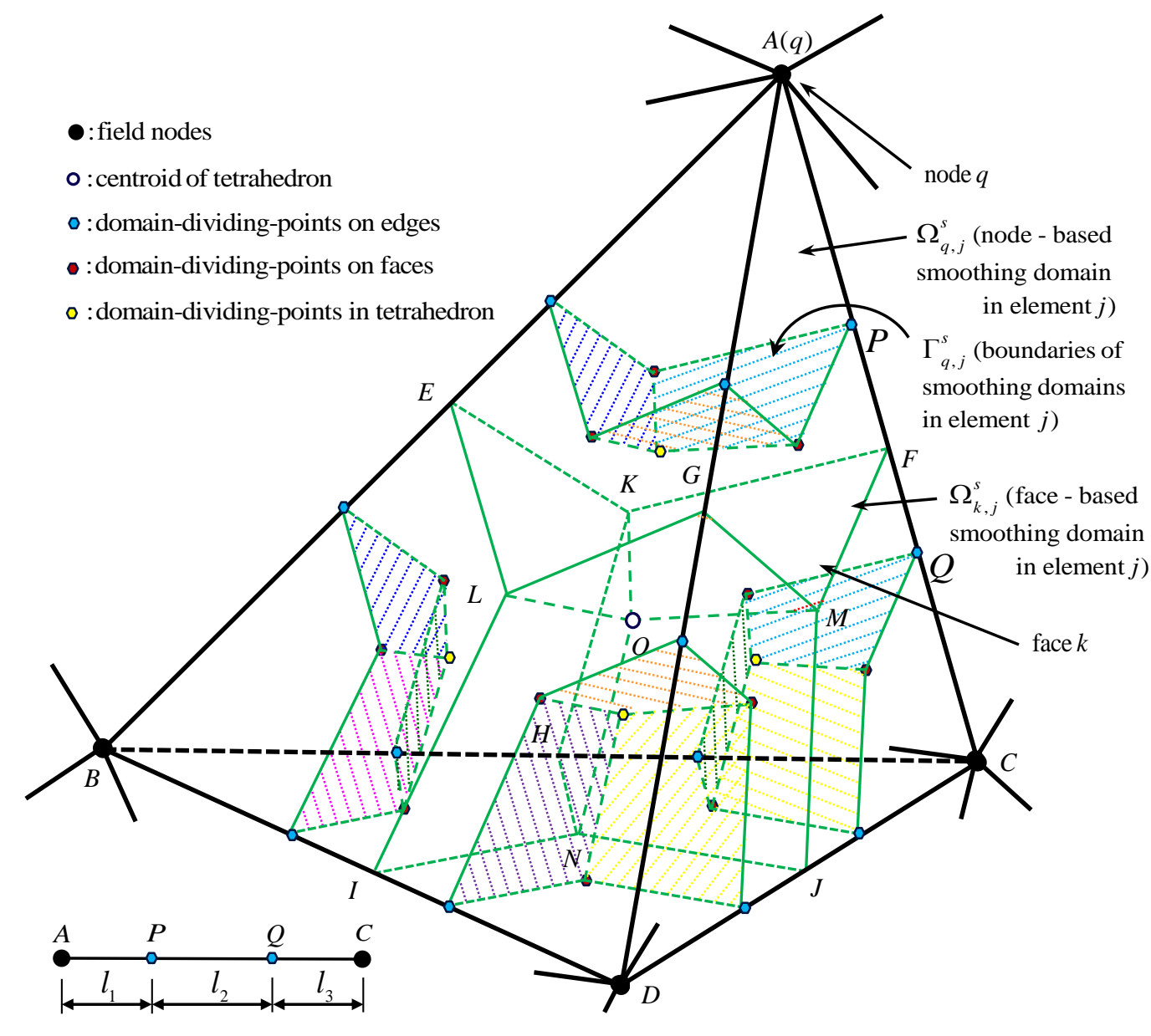

Fig. 4. Scheme of smoothing domains in a representative element for $\beta$ FEM-T4: the node-based sub-smoothing domains and face-based sub-smoothing domains.

It is noticed that $\beta \mathrm{FEM}$ using $\mathrm{T}$-elements can be regarded as a combination of the features from both the NS-FEM and ES/FS-FEM, since the smoothing domains are established based on both node-based and edge/face-based smoothing techniques.

Here we can choose $(\bar{\bullet}),(\tilde{\bullet})$ and $(\boldsymbol{\bullet})$ to denote the physical quantities obtained from the NS-FEM, ES/FS-FEM and $\beta$ FEM respectively. For 2D problems, the area of smoothing domain for $\beta$ FEM $\left(\widehat{A}^{s}\right)$ is comprised by the area of the node-based smoothing domain $\left(\bar{A}^{s}\right)$ and edge-based smoothing domain $\left(\tilde{A}^{s}\right)$ : 


$$
\widehat{A}^{s}=\bar{A}^{s}+\tilde{A}^{s}
$$

with

$$
\bar{A}^{s}=\beta^{2} \widehat{A}^{s} \quad \text { and } \quad \tilde{A}^{s}=\left(1-\beta^{2}\right) \widehat{A}^{s}, \quad \beta \in[0,1]
$$

For 3-D problems, Eq. (20) and (21) can be expressed in forms as

$$
\begin{gathered}
\hat{V}^{s}=\bar{V}^{s}+\tilde{V}^{s} \\
\bar{V}^{s}=\beta^{3} \widehat{V}^{s} \quad \text { and } \quad \tilde{V}^{s}=\left(1-\beta^{3}\right) \widehat{V}^{s}, \beta \in[0,1]
\end{gathered}
$$

where $\widehat{V}^{s}$ denotes the volume of the smoothing domain for 3-D problems.

In a $\beta$ FEM scheme, the smoothed strain-displacement matrix $\overline{\mathbf{B}}_{I}$ for $\Omega_{q}^{s}$ is defined as

$$
\overline{\mathbf{B}}_{I}=\frac{1}{A_{k}^{s}} \sum_{j=1}^{n_{k}^{e}} \frac{1}{3} \beta^{2} A_{j}^{e} \mathbf{B}_{j}^{e} \quad \text { or } \quad \overline{\mathbf{B}}_{I}=\frac{1}{V_{k}^{s}} \sum_{j=1}^{n_{k}^{e}} \frac{1}{4} \beta^{3} V_{j}^{e} \mathbf{B}_{j}^{e}
$$

The smoothed strain-displacement matrix $\tilde{\mathbf{B}}_{I}$ for $\Omega_{k}^{s}$ will be of the form

$$
\tilde{\mathbf{B}}_{I}=\frac{1}{A_{q}^{s}} \sum_{l=1}^{n_{q}^{e}} \frac{1}{3}\left(1-\beta^{2}\right) A_{l}^{e} \mathbf{B}_{l}^{e} \text { or } \quad \tilde{\mathbf{B}}_{I}=\frac{1}{V_{q}^{s}} \sum_{l=1}^{n_{q}^{e}} \frac{1}{4}\left(1-\beta^{3}\right) V_{l}^{e} \mathbf{B}_{l}^{e}
$$

Now we can obtain the smoothed stiffness matrix $\overline{\mathbf{K}}_{I J}^{(q)}$ for smoothing domain $\Omega_{q}^{s}$, which gives

$$
\overline{\mathbf{K}}_{I J}^{(q)}=\int_{\Omega_{q}^{s}} \overline{\mathbf{B}}_{I}^{T} \mathbf{D} \overline{\mathbf{B}}_{J} \mathrm{~d} \Omega=\beta^{2} \overline{\mathbf{B}}_{I}^{T} \mathbf{D} \overline{\mathbf{B}}_{J} A_{q}^{s} \text { or } \quad \overline{\mathbf{K}}_{I J}^{(q)}=\int_{\Omega_{q}^{s}} \overline{\mathbf{B}}_{I}^{T} \overline{\mathbf{D}}_{J} \mathrm{~d} \Omega=\beta^{3} \overline{\mathbf{B}}_{I}^{T} \mathbf{D} \overline{\mathbf{B}}_{J} V_{q}^{s}
$$

For edge-based smoothing domain $\Omega_{k}^{s}$, the smoothed stiffness matrix $\tilde{\mathbf{K}}_{I J}^{(k)}$ will be obtained by a similar fashion as follows

$$
\begin{gathered}
\tilde{\mathbf{K}}_{I J}^{(k)}=\int_{\Omega_{k}^{s}} \tilde{\mathbf{B}}_{I}^{T} \mathbf{D} \tilde{\mathbf{B}}_{J} \mathrm{~d} \Omega=\left(1-\beta^{2}\right) \tilde{\mathbf{B}}_{I}^{T} \mathbf{D} \tilde{\mathbf{B}}_{J} A_{k}^{s} \text { or } \\
\tilde{\mathbf{K}}_{I J}^{(k)}=\int_{\Omega_{k}^{s}} \tilde{\mathbf{B}}_{I}^{T} \mathbf{D} \tilde{\mathbf{B}}_{J} \mathrm{~d} \Omega=\left(1-\beta^{3}\right) \tilde{\mathbf{B}}_{I}^{T} \mathbf{D} \tilde{\mathbf{B}}_{J} V_{k}^{s}
\end{gathered}
$$

Since the portion of the area/volume of node-based and edge/face-based smoothing domains (or NS-FEM and ES/FS-FEM) will be tuned by $\beta$, the global stiffness matrix 
will be assembled by the contributions from both of them. Therefore the global stiffness matrix $\widehat{\mathbf{K}}$ for $\beta$ FEM can be assembled from the $\overline{\mathbf{K}}_{I J}^{(q)}$ and $\tilde{\mathbf{K}}_{I J}^{(k)}$ as follows

$$
\widehat{\mathbf{K}}=\sum_{q=1}^{N_{n}} \overline{\mathbf{K}}_{I J}^{(q)}+\sum_{k=1}^{N_{e}} \tilde{\mathbf{K}}_{I J}^{(k)}
$$

where $N_{e}$ and $N_{n}$ are the number of total edges/faces and total nodes in the system.

\subsection{Properties of $\beta F E M$}

The properties of ES/FS-FEM and NS-FEM, including the variational consistence, displacement compatibility, and solution continuity, have been studies and summarized in [24,32-34]. Since the continuous scalar factor $\beta$ for $\beta$ FEM can be regarded as a knob which controls the contributions from the NS-FEM and ES/FS-FEM, it indicates that we can obtain a continuous solution function from the solution of the ES/FS-FEM to that of NS-FEM if the factor $\beta$ turns from 0 to 1 . Therefore, the $\beta$ FEM shall possess some properties of both NS-FEM and ES/FS-FEM due to this fact. If we take an elastic static problem as our example and the strain field of an elastic problem obtained by NS-FEM and ES/FS-FEM is assumed as $\overline{\boldsymbol{\varepsilon}}$ and $\tilde{\boldsymbol{\varepsilon}}$ respectively, we can obtain the potential energy functional of $\beta$ FEM based on the virtual work principle similar as the standard FEM:

$$
\widehat{\Pi}(\hat{\mathbf{u}}, \beta)=\widehat{\Pi}^{\mathrm{int}}(\hat{\mathbf{u}})-\widehat{\Pi}^{\mathrm{ext}}(\hat{\mathbf{u}})=\bar{\Pi}^{\mathrm{int}}(\hat{\mathbf{u}})+\tilde{\Pi}^{\mathrm{int}}(\hat{\mathbf{u}})-\widehat{\Pi}^{\mathrm{ext}}(\hat{\mathbf{u}})
$$

For the problem using isotropic linear elastic material, the Eq. (29) becomes

$$
\widehat{\Pi}(\hat{\mathbf{u}}, \beta)=\int_{\Omega} \frac{1}{2} \overline{\boldsymbol{\varepsilon}}^{\mathrm{T}}(\hat{\mathbf{u}}) \mathbf{D} \overline{\boldsymbol{\varepsilon}}(\hat{\mathbf{u}}) \mathrm{d} \Omega+\int_{\Omega} \frac{1}{2} \tilde{\boldsymbol{\varepsilon}}^{\mathrm{T}}(\hat{\mathbf{u}}) \mathbf{D} \tilde{\boldsymbol{\varepsilon}}(\hat{\mathbf{u}}) \mathrm{d} \Omega-\int_{\Omega} \mathbf{f}^{b} \cdot \hat{\mathbf{u}} \mathrm{d} \Omega-\int_{\Gamma_{t}} \mathbf{f}^{t} \cdot \hat{\mathbf{u}} \mathrm{d} \Gamma
$$

where the given continuous scalar factor should be $\beta \in[0,1], \hat{\mathbf{u}}$ is an admissible virtual displacement field which satisfies the given essential boundary conditions, and D 
denotes the elasticity tensor. If one performs variation to $\hat{\mathbf{u}}$ by the chain rule, the above equation will be written as

$$
\delta \widehat{\Pi}(\hat{\mathbf{u}}, \beta)=\delta \int_{\Omega} \overline{\boldsymbol{\varepsilon}}^{\mathrm{T}}(\hat{\mathbf{u}}) \mathbf{D} \overline{\boldsymbol{\varepsilon}}(\hat{\mathbf{u}}) \mathrm{d} \Omega+\delta \int_{\Omega} \tilde{\boldsymbol{\varepsilon}}^{\mathrm{T}}(\hat{\mathbf{u}}) \mathbf{D} \tilde{\boldsymbol{\varepsilon}}(\hat{\mathbf{u}}) \mathrm{d} \Omega-\int_{\Omega} \delta \hat{\mathbf{u}} \mathbf{f}^{b} \mathrm{~d} \Omega-\int_{\Gamma_{t}} \delta \hat{\mathbf{u}}^{t} \mathrm{~d} \Gamma
$$

Several important properties can be found through examining the above equations, as follows:

Property 1 (variational consistence). The $\beta$ FEM is variational consistent for both $2 \mathrm{D}$ and 3D problems.

For a problem domain $\Omega$, it can be discretized into edge/face-based smoothing cells $\Omega_{k}^{s}$ associated with $N_{e d}$ (or $N_{\text {face }}$ ) edges/faces and node-based smoothing cells $\Omega_{q}^{s}$ associated with $N_{n}$ nodes. If we substitute the approximation (3) and (9) (similar form for node-based smoothing cells) into Eq. (31) and utilize the arbitrary property of variation, the equation of interest for an element can be obtained as following

$$
\mathbf{K}^{\text {two-field }} \mathbf{d}=\mathbf{f}
$$

where $\mathbf{f}$ is the force vector and $\mathbf{K}^{\text {two-field }}$ denotes the smoothed stiffness matrix leading to

$$
\mathbf{K}^{\text {two-field }}=\int_{\Omega_{q}^{s}} \overline{\mathbf{B}}_{I}^{T} \mathbf{D} \overline{\mathbf{B}}_{J} \mathrm{~d} \Omega+\int_{\Omega_{k}^{s}} \tilde{\mathbf{B}}_{I}^{T} \mathbf{D} \tilde{\mathbf{B}}_{J} \mathrm{~d} \Omega
$$

Noted that the first term follows NS-FEM and second term follows ES/FS-FEM, and both of them are variationally consistent [13].

Property 2 (bound property). The solution of $\beta$ FEM is bounded by the corresponding NS-FEM and ES/FS-FEM.

For the factor $\beta=1$, the $\beta$ FEM scheme becomes the standard NS-FEM, which possesses the property of underestimation of strain energy and stiffness values as reported $[28,36]$. The upper bound property is then ensured, which will be confirmed in numerical examples in Section 6. 
When we choose $\beta=0$, the $\beta$ FEM is essentially the same as the ES/FS-FEM. The stiffness will be overestimated [26, 32, 38-40], although it has higher accuracy compared to standard FEM. This leads to the low bound property of $\beta$ FEM.

It is important to note that the solution of $\beta$ FEM will be within a narrow interval, which has a lower bound of solution generated by ES/FS-FEM and a upper bound of solution generated by NS-FEM. Moreover, this interval will be narrower than $\alpha$ FEM [37], since compared to standard FEM the ES/FS-FEM produces more accurate solution from the lower bound;

Property 3 (solution continuity property). If the scaling factor $\beta$ varies from 0.0 to 1.0 , the overestimation property of stiffness becomes underestimation, continuously, and the solution of $\beta$ FEM will be a continuous function of $\beta$ from the solution of the ES/FS-FEM to that of NS-FEM.

Property 4 (exact solution property). According to the solution continuity property [24], the exact or close-to-exact solution(s) of strain energy can be approached by tuning the scalar factor $\beta$.

Property 5 (temporal stability property). Even with a small portion of ES/FS-FEM (choosing a value of $\beta$ close to 1), the constructed stiffness matrix exhibits the properties of overestimation, which can alleviate the temporal instability existed in the pure NS-FEM.

\section{Implementation aspects}

The section will briefly present the implementation aspects related to the numerical examples, including the formulations for free vibration analysis and large deformation problems. 


\subsection{Formulations for free vibration problem}

The general discrete form of Eq. (1) for vibration analysis using $\beta$ FEM gives the form as

$$
\mathbf{M} \ddot{\mathbf{d}}+\mathbf{C} \dot{\mathbf{d}}+\hat{\mathbf{K}} \mathbf{d}=\mathbf{f}
$$

where $\mathbf{M}, \mathbf{C}$ and $\hat{\mathbf{K}}$ are the matrices of mass, damping and stiffness. If the terms of damping and external forces are not considered, the above equation can be simplified into a free vibration problem with a homogenous form:

$$
\mathbf{M} \ddot{\mathbf{d}}+\widehat{K} \mathbf{d}=\mathbf{0}
$$

where the it can adopt lumped mass matrix or consistent mass matrix for $\mathbf{M}$. The general solution of Eq. (35) can be assumed as [41]

$$
\mathbf{d}=\varphi \exp (\mathrm{i} \omega t)
$$

in which the eigenvector $\varphi$ and natural frequency $\omega$ can be determined by the eigenvalue equation as following:

$$
(-\Lambda \mathbf{M}+\widehat{\mathbf{K}}) \psi=0
$$

and

$$
\begin{gathered}
\Lambda=\operatorname{diagonal}\left(\omega_{1}^{2}, \omega_{2}^{2}, \ldots, \omega_{n}^{2}\right)=\operatorname{diagonal}\left(\lambda_{1}, \lambda_{2}, \ldots, \lambda_{n}\right) \\
\boldsymbol{\psi}=\left[\boldsymbol{\varphi}_{1}, \boldsymbol{\varphi}_{2}, \ldots, \boldsymbol{\varphi}_{n}\right]
\end{gathered}
$$

where $\varphi_{r}$ is the eigenvector associated with the eigenvalue $\lambda_{r} \quad(r=1,2 \ldots, n)$.

We can also define modal stiffness $k_{r}$ and modal mass $m_{r}$ of the system as

$$
\begin{aligned}
& k_{r}=\boldsymbol{\varphi}_{r}^{T} \mathbf{K} \boldsymbol{\varphi}_{r}, \quad r=1,2 \ldots, n \\
& m_{r}=\varphi_{r}^{T} \mathbf{M} \boldsymbol{\varphi}_{r}, \quad r=1,2 \ldots, n
\end{aligned}
$$

In this case the natural frequency can now be evaluated using the following relationship:

$$
\omega_{r}=\sqrt{k_{r} / m_{r}}, \quad r=1,2 \ldots, n
$$




\subsection{Nonlinear problems with large deformation}

For nonlinear problem with large deformation, it can be solved by an incremental process. If we introduce the tangent stiffness matrix ${ }^{t} \mathbf{K}$ and internal force vector $\mathbf{f}^{I}$, the FEM equilibrium equation of large deformation according to total Lagrange formulation $[24,38-40]$ becomes

$$
{ }^{t} \mathbf{K d}=\mathbf{f}^{R}=\mathbf{f}^{E}-\mathbf{f}^{I}
$$

In finite element computations, the tangent stiffness matrix is frequently split into linear part ${ }^{t} \mathbf{K}_{L}$ and nonlinear part ${ }^{t} \mathbf{K}_{N L}$, then we can rewrite the above equation using $\beta$ FEM as

$$
\left({ }^{t} \widehat{\mathbf{K}}_{L}+{ }^{t} \widehat{\mathbf{K}}_{N L}\right) \mathbf{d}=\mathbf{f}^{E}-\widehat{\mathbf{f}}^{I}
$$

where the linear part

$$
{ }^{t} \widehat{\mathbf{K}}_{L}=\sum_{q=1}^{N_{n}} \beta^{2} \overline{\mathbf{B}}_{L}^{T} \mathbf{D} \overline{\mathbf{B}}_{L} V_{q}^{s}+\sum_{k=1}^{N_{e}}\left(1-\beta^{2}\right) \tilde{\mathbf{B}}_{L}^{T} \mathbf{D} \tilde{\mathbf{B}}_{L} V_{k}^{s}
$$

the contribution, ${ }^{t} \hat{\mathbf{K}}_{N L}$, is defined as

$$
{ }^{t} \widehat{\mathbf{K}}_{N L}=\sum_{q=1}^{N_{n}} \beta^{2} \overline{\mathbf{B}}_{N L}^{T} \overline{\mathbf{S}} \overline{\mathbf{B}}_{N L} V_{q}^{s}+\sum_{k=1}^{N_{e}}\left(1-\beta^{2}\right) \tilde{\mathbf{B}}_{N L}^{T} \tilde{\mathbf{S}} \tilde{\mathbf{B}}_{N L} V_{k}^{s}
$$

and the internal force vector reads

$$
\widehat{\mathbf{f}}^{I}=\sum_{q=1}^{N_{n}} \beta^{2} \overline{\mathbf{B}}_{L}^{T}\{\overline{\mathbf{S}}\} V_{q}^{s}+\sum_{k=1}^{N_{e}}\left(1-\beta^{2}\right) \tilde{\mathbf{B}}_{L}^{T}\{\tilde{\mathbf{S}}\} V_{k}^{s}
$$

where the matrix $\overline{\mathbf{B}}_{L}, \tilde{\mathbf{B}}_{L}, \overline{\mathbf{B}}_{N L}, \tilde{\mathbf{B}}_{N L}, \overline{\mathbf{S}}, \tilde{\mathbf{S}},\{\overline{\mathbf{S}}\}$ and $\{\tilde{\mathbf{S}}\}$ can be smoothed from $\mathbf{B}_{L}, \mathbf{B}_{N L}, \mathbf{S}$ and $\{\mathbf{S}\}$ via a same fashion presented in Section 2. The expressions of these matrices $\left(\mathbf{B}_{L}, \mathbf{B}_{N L}, \mathbf{S}\right.$ and $\left.\{\mathbf{S}\}\right)$ for 3D problems are given as (2D expressions will be easily obtained [24]) 


$$
\begin{aligned}
& \mathbf{B}_{L}=\left[\begin{array}{ccc}
F_{11} N_{1,1} & F_{21} N_{1,1} & F_{31} N_{1,1} \\
F_{12} N_{1,2} & F_{22} N_{1,2} & F_{32} N_{1,2} \\
F_{13} N_{1,3} & F_{23} N_{1,3} & F_{33} N_{1,3} \\
F_{11} N_{1,2}+F_{12} N_{1,1} & F_{21} N_{1,2}+F_{22} N_{1,1} & F_{31} N_{1,2}+F_{32} N_{1,1} \\
F_{12} N_{1,3}+F_{13} N_{1,2} & F_{22} N_{1,3}+F_{23} N_{1,2} & F_{32} N_{1,3}+F_{33} N_{1,2} \\
F_{13} N_{1,1}+F_{11} N_{1,3} & F_{23} N_{1,1}+F_{21} N_{1,3} & F_{33} N_{1,1}+F_{31} N_{1,3}
\end{array}\right. \\
& \begin{array}{lll}
F_{11} N_{1,1} & \ldots & F_{31} N_{4,1}
\end{array} \\
& F_{12} N_{1,2} \quad \ldots \quad F_{32} N_{4,2} \\
& \begin{array}{lll}
F_{13} N_{1,3} & \cdots & F_{33} N_{4,3}
\end{array} \\
& F_{11} N_{1,2}+F_{12} N_{1,1} \quad \ldots \quad F_{31} N_{4,2}+F_{32} N_{4,1} \\
& F_{12} N_{1,3}+F_{13} N_{1,2} \quad \ldots \quad F_{32} N_{4,3}+F_{33} N_{4,2} \\
& \left.F_{13} N_{1,1}+F_{11} N_{1,3} \quad \ldots \quad F_{33} N_{4,1}+F_{31} N_{4,3}\right]
\end{aligned}
$$

where the deformation gradient tensor $\mathbf{F}$ is defined by

$$
\mathbf{F}=\left(\frac{\partial \mathbf{x}}{\partial \mathbf{X}}\right)^{T}=\left[\begin{array}{lll}
F_{11} & F_{12} & F_{13} \\
F_{21} & F_{22} & F_{23} \\
F_{31} & F_{32} & F_{33}
\end{array}\right]
$$

The matrices $\mathbf{B}_{N L}$ and $\mathbf{S}$ are given by

$$
\mathbf{B}_{N L}=\left[\begin{array}{cccccc}
N_{1,1} & 0 & 0 & N_{2,1} & \ldots & 0 \\
N_{1,2} & 0 & 0 & N_{2,2} & \ldots & 0 \\
N_{1,3} & 0 & 0 & N_{2,3} & \ldots & 0 \\
0 & N_{1,1} & 0 & 0 & \ldots & 0 \\
0 & N_{1,2} & 0 & 0 & \ldots & 0 \\
0 & N_{1,3} & 0 & 0 & \ldots & 0 \\
0 & 0 & N_{1,1} & 0 & \ldots & N_{4,1} \\
0 & 0 & N_{1,2} & 0 & \ldots & N_{4,2} \\
0 & 0 & N_{1,3} & 0 & \ldots & N_{4,3}
\end{array}\right]
$$

and 


$$
\mathbf{S}=\left[\begin{array}{ccccccccc}
S_{11} & S_{12} & S_{13} & 0 & 0 & 0 & 0 & 0 & 0 \\
S_{12} & S_{22} & S_{23} & 0 & 0 & 0 & 0 & 0 & 0 \\
S_{13} & S_{23} & S_{33} & 0 & 0 & 0 & 0 & 0 & 0 \\
0 & 0 & 0 & S_{11} & S_{12} & S_{13} & 0 & 0 & 0 \\
0 & 0 & 0 & S_{12} & S_{22} & S_{23} & 0 & 0 & 0 \\
0 & 0 & 0 & S_{13} & S_{23} & S_{33} & 0 & 0 & 0 \\
0 & 0 & 0 & 0 & 0 & 0 & S_{11} & S_{12} & S_{13} \\
0 & 0 & 0 & 0 & 0 & 0 & S_{12} & S_{22} & S_{23} \\
0 & 0 & 0 & 0 & 0 & 0 & S_{13} & S_{23} & S_{33}
\end{array}\right]
$$

The second Piola-Kirchhoff stress tensor (PK2) holds the form as

$$
\{\mathbf{S}\}=\left\lfloor\begin{array}{llllll}
S_{11} & S_{22} & S_{33} & S_{12} & S_{23} & S_{31}
\end{array}\right\rfloor^{T}=\mathbf{D}\left\lfloor\begin{array}{llllll}
E_{11} & E_{22} & E_{33} & 2 E_{12} & 2 E_{23} & 2 E_{31}
\end{array}\right\rfloor^{T}
$$

where the Green-Lagrange strain tensor $\mathbf{E}$ of elements can be defined from the deformation gradient tensor, which reads

$$
\mathbf{E}=\frac{1}{2}\left(\mathbf{F}^{T} \mathbf{F}-\mathbf{I}\right)=\left[\begin{array}{lll}
E_{11} & E_{12} & E_{13} \\
E_{21} & E_{22} & E_{23} \\
E_{31} & E_{32} & E_{33}
\end{array}\right]
$$

\section{Standard patch test}

\subsection{A standard patch test for $2 D$ problems}

To validate our presented $\beta$ FEM, the satisfaction of patch tests is an essential requirement. In Fig. 5, a simple domain is discretized using a few pieces of "patch" with irregular triangular elements indicated by red color. The left and bottom edges are constrained along the horizontal and vertical directions, respectively. The right and top edges are assumed to be stretched to $10 \%$ of the original length along the horizontal and vertical directions, respectively. In order to pass the patch test, the evaluated displacements of all the interior nodes shall follow exactly (to machine precision) as the same linear function of the displacements applied along the edges, viz., 


$$
u=0.1 x \quad \text { and } \quad v=0.1 y
$$

To examine the numerical convergence rate, the following displacement error norm can be defined

$$
e_{d}=\frac{\sum_{i=1}^{\text {ndof }}\left|u_{i}-u_{i}^{h}\right|}{\sum_{i=1}^{\text {ndof }}\left|u_{i}\right|} \times 100 \%
$$

where $u_{i}$ and $u_{i}^{h}$ are the exact and numerical solution of displacements, respectively.

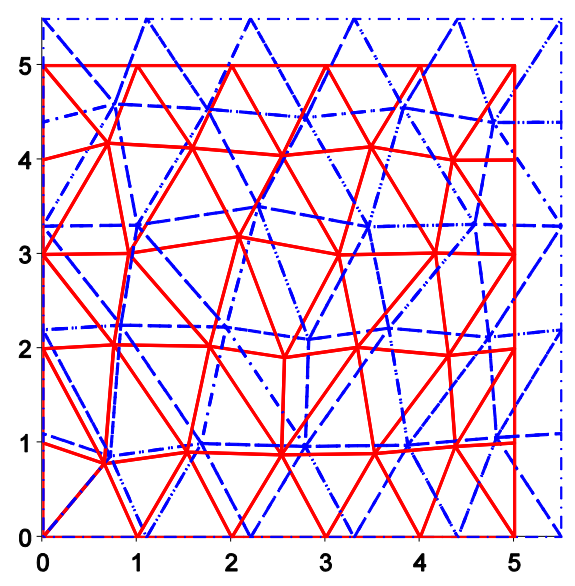

Fig. 5. A 2D patch test for $\beta$ FEM using triangular mesh.

The deformed configuration is displayed by blue dash-dot lines in Fig. 5. In Table 1, the numerical results show that this simple square model is able to pass the designed patch test within machine precision for all the values $\beta \in[0,1]$. Thus, the displacement compatibility is ensured and the convergence of numerical solutions is guaranteed.

Table 1 Displacement error norm for 2D patch test

\begin{tabular}{cccccccc}
\hline$\beta$ & 0.0000 & 0.2000 & $0.4853^{*}$ & $0.6324^{*}$ & 0.8000 & 0.9000 & 1.0000 \\
\hline$e_{d}$ & $2.7933 \mathrm{e}-14$ & $2.8501 \mathrm{e}-14$ & $2.9852 \mathrm{e}-14$ & $3.6817 \mathrm{e}-14$ & $2.7293 \mathrm{e}-14$ & $3.5822 \mathrm{e}-14$ & $5.0748 \mathrm{e}-14$ \\
\hline
\end{tabular}

* Random number 


\subsection{Irons first-order patch test for $3 D$ problems}

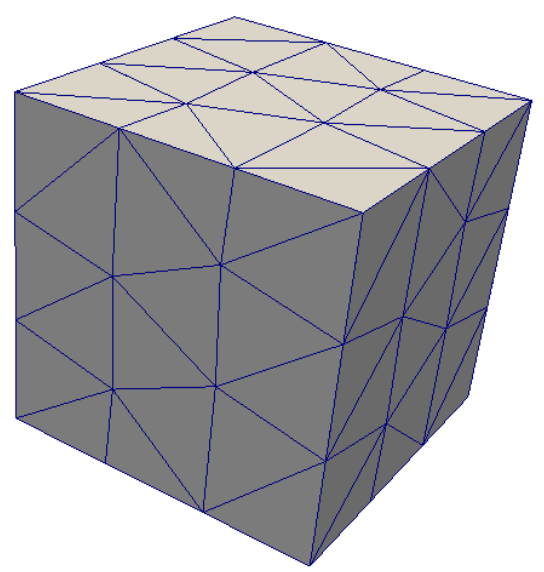

Fig. 6. A $3 D$ cubic patch test for $\beta$ FEM using tetrahedral mesh.

Now we consider a 3D elastic cube with side length $10 \mathrm{~mm}$ and material parameters $E=6.895 \times 10^{3} \mathrm{MPa}$ and $v=0.25$. On the exterior boundaries, linear displacements are prescribed as following:

$$
\begin{gathered}
u=0.0005^{*}(2 x+y+z) \\
v=0.0005^{*}(x+2 y+z) \\
w=0.0005^{*}(x+y+2 z)
\end{gathered}
$$

The domain needs to be discretized by irregular elements with at least one interior node, e.g., Fig. 6. To pass this patch test, the displacements of all the interior nodes should follow exactly the same function of the imposed displacement on exterior boundaries. The displacement error norms calculated by Eq. (55) are listed in Table 2. Again it passed the conducted patch test at machine precision and the displacement compatibility can be ensured.

Table 2 Displacement error norm for 3D patch test

\begin{tabular}{c|c|c|c|c|c|c|c}
\hline$\beta$ & 0.0000 & 0.2000 & $0.4218^{*}$ & $0.6555^{*}$ & 0.7500 & 0.9000 & 1.0000 \\
\hline$e_{d}$ & $7.1012 \mathrm{e}-16$ & $3.0434 \mathrm{e}-16$ & $6.0868 \mathrm{e}-16$ & $6.0868 \mathrm{e}-16$ & $7.1012 \mathrm{e}-16$ & $4.0579 \mathrm{e}-16$ & $8.1157-16$ \\
\hline
\end{tabular}

* Random number 


\section{Numerical examples and discussion}

In this section, several representative numerical examples are illustrated. In the first example, the well-known Cook's membrane problem under plane stress condition is studied for the comparisons of accuracy and solution bounds. An infinite elastic plate with a circular hole under remote tensile load is considered as a plane strain problem to test the accuracy and simple volumetric locking in the second example. The third example tests the property of temporal stability of $\beta$ FEM by analysis of free vibration of an automobile connecting bar. The fourth and fifth examples examine the accuracy of proposed 3D $\beta$ FEM. A human molar tooth example is simulated in the sixth example, which shows the application of our method for modeling of an object with moderately complex geometry. The last example extends the method to geometrically non-linear analysis of large deformation.

\subsection{Cook's membrane: study of accuracy and solution bounds}

As a standard test for combined bending and shear response with moderate distortion, Cook's membrane problem [42] is shown in Fig. 7. The problem consists of a tapered panel clamped at the left boundary and subjected to an in-plane shearing traction at the free right edge. The volume force would not be considered and the plane stress conditions are assumed. The material parameters are chosen as: Young's modulus $E=3 \times 10^{7} \mathrm{~Pa}$ and Poisson's ratio $v=1 / 3$. According to References $[7,43]$, we set the

geometrical dimensions as $l_{1}=48, l_{2}=44$ and $l_{3}=16$, and the loading $P=1$, where $P$ is the resultant of the uniformly distributed shear traction. The reference value of the vertical displacement for the middle of right edge is 23.9642 [44] and the reference strain energy of membrane is 12.015 [42]. 


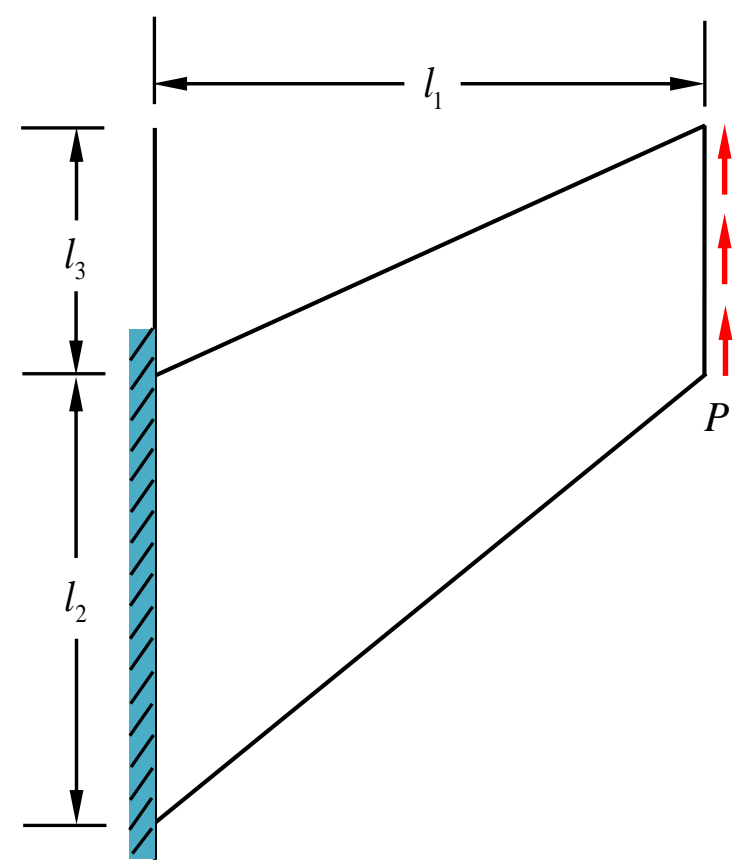

(a)

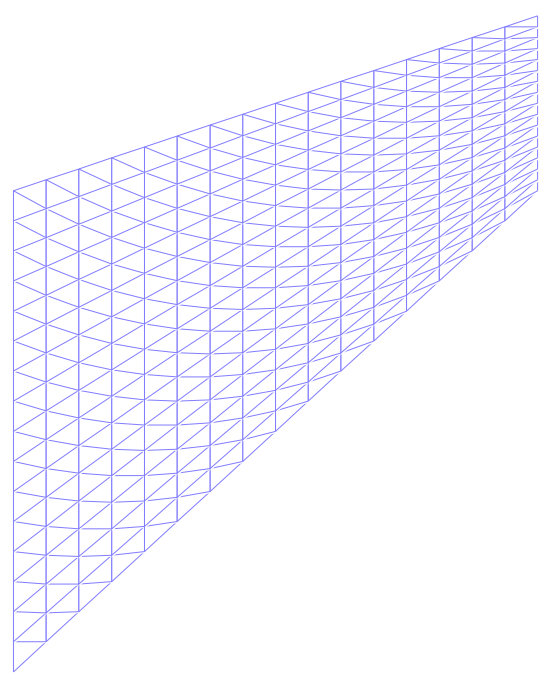

(b)

Fig. 7. Cook's membrane problem: (a) geometry and loads and (b) domain discretization by $16 \times 16$ triangular base mesh.

In order to test the accuracy and convergence behavior of the $\beta$ FEM, the problem has been discretized into structured meshes with $N \times N$ edge density of mesh. Fig. 8 and Fig. 9 compare the results obtained by several different methods with four different spatial discretizations. Fig. 8 shows comparisons of displacements at right tip-center of the membrane computed by different methods. The bound properties of strain energy are investigated and compared in Fig. 9. It is evident that the $\beta$ FEM (with $\beta=0.5$ ) generates the most accurate solutions (or close-to-reference solutions) among all these methods. The FEM and ES-FEM produce stiffer solutions of displacement and show the overestimation property of stiffness, which approximates the reference solutions from the lower-bound of displacement or energy. On the other hand, the NS-FEM produces "overly-soft" solutions because of the underestimation behavior, which reflects the 
unique property of upper-bound. Regardless of the value of parameter $\beta$, the numerical results of $\beta$ FEM should be within the narrow interval bounded by the solutions of ES-FEM and NS-FEM.

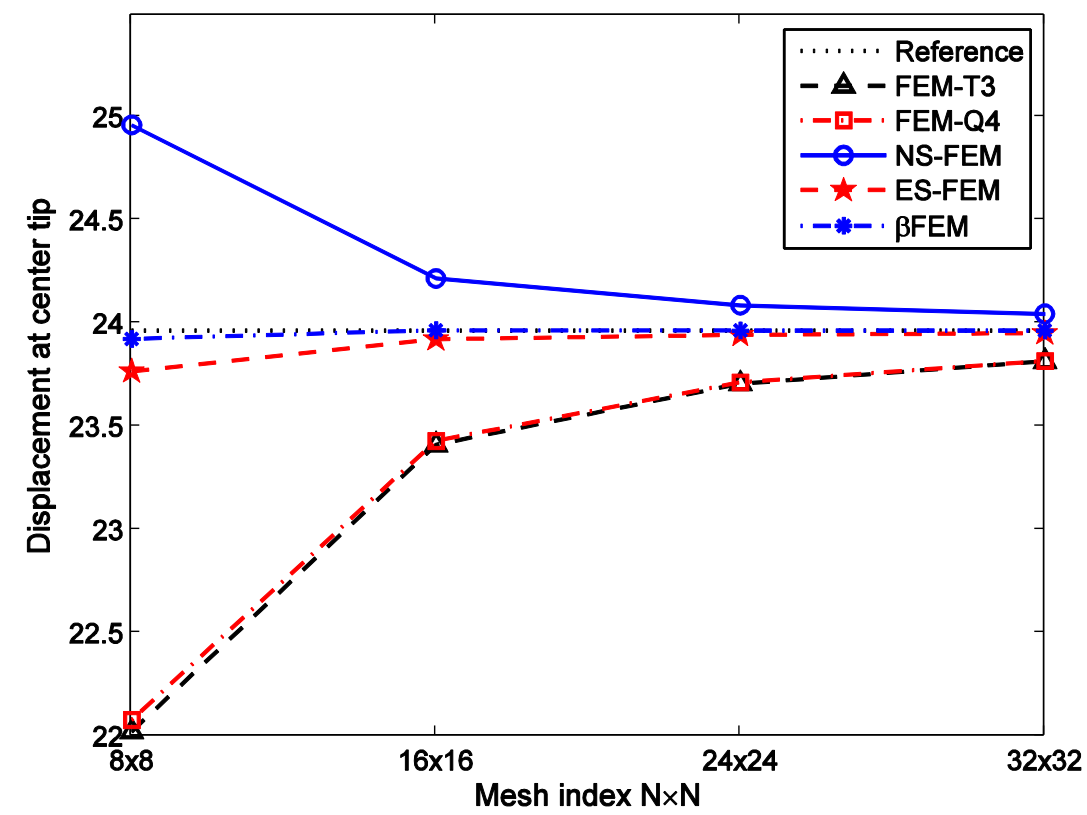

Fig. 8. Comparisons of displacements at tip-center from different methods for Cook's membrane problem. 


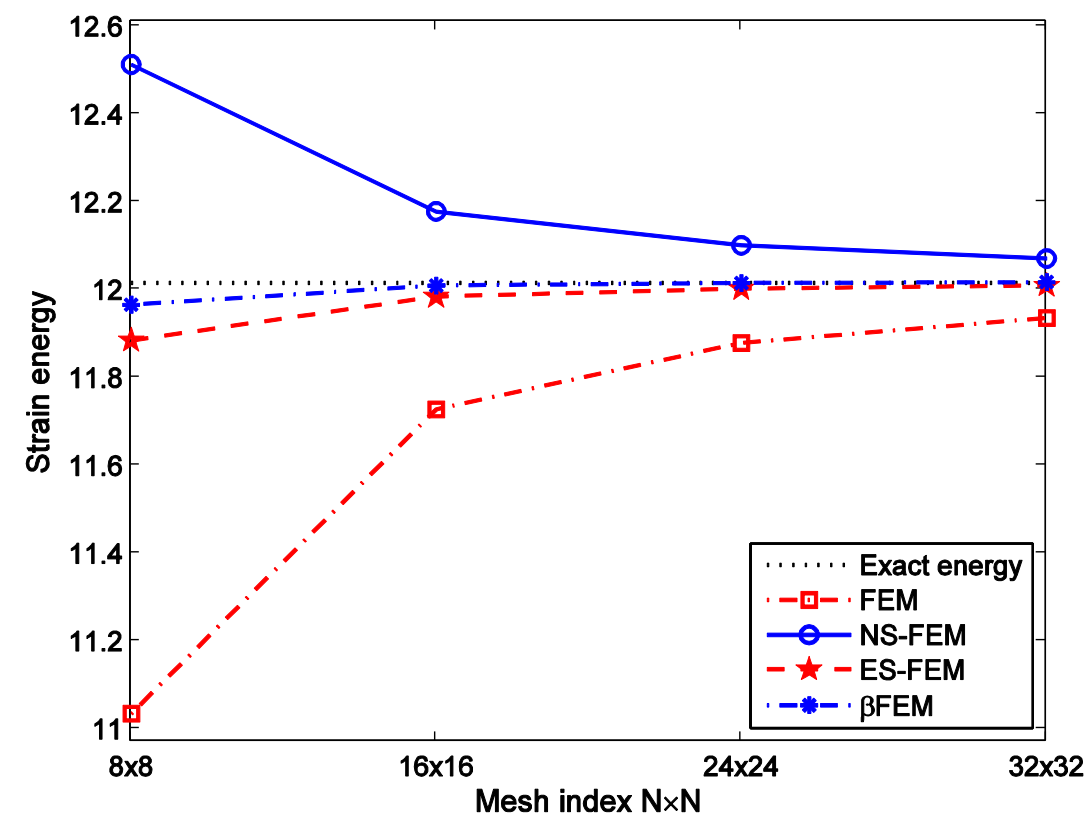

Fig. 9. Solution bounds of energy for Cook's membrane problem.

6.2 Circular hole in an infinite plate under remote tensile load: test for accuracy and volumetric locking
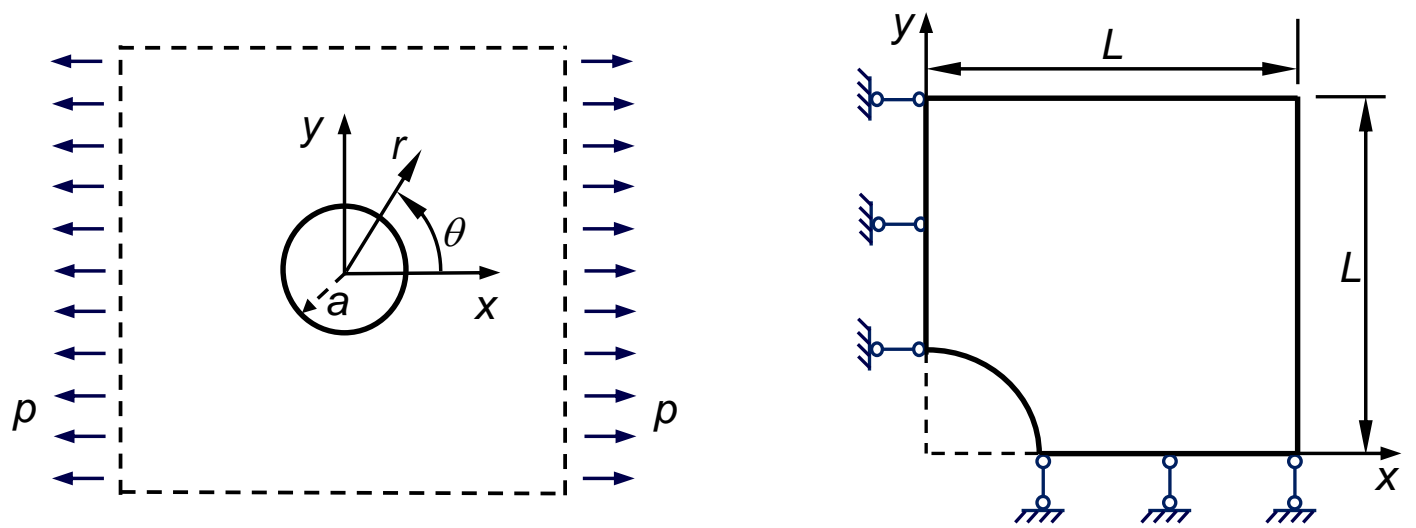

Fig. 10. Circular hole in an infinite plate under tension and its quarter model.

Consider the infinite plate with a central circular hole as shown in Fig. 10, loaded in tension by a force per unit area, $p=0.98 \times 10^{6} \mathrm{~N} / \mathrm{m}^{2}$, in the $\mathrm{x}$ direction. Since the stress concentration decreases very rapidly if the distance to the center increases, the infinite 
plate problem can be treated as an equivalent finite plate problem, i.e., a finite plate with $L=5 a$ under the same loading/boundary conditions. Due to the symmetry of problem, only one quarter (upper right quadrant) of the plate is necessary to be modeled and discretized into T3 or Q4 elements. We can assume the plane strain condition and the geometrical parameters are set as $a=0.2 \mathrm{~m}$ and $L=1.0 \mathrm{~m}$. The exact solution for displacement components is given by [45]

$$
\begin{aligned}
& u_{x}=\frac{p a}{8 \mu}\left[\left(2 \frac{a}{r}+\frac{r}{a}\right)(1+\kappa) \cos \theta+2 \frac{a}{r}\left(1-\frac{a^{2}}{r^{2}}\right) \cos 3 \theta\right] \\
& u_{y}=\frac{p a}{8 \mu}\left[\left(2 \frac{a}{r}-\frac{r}{a}\right)(1-\kappa) \sin \theta+2 \frac{a}{r}\left(1-\frac{a^{2}}{r^{2}}\right) \sin 3 \theta\right]
\end{aligned}
$$

where $(r, \theta)$ stands for the polar coordinates with counterclockwise measure $\theta$, and for the plane strain condition we have the shear modulus $\mu=E /(2(1+v))$ and bulk modulus $\kappa=3-4 v$. The overall stress distributions in the plate are given by [45]

$$
\begin{aligned}
& \sigma_{11}=p\left[1-\frac{a^{2}}{r^{2}}\left(\frac{3}{2} \cos 2 \theta+\cos 4 \theta\right)+\frac{3 a^{4}}{2 r^{4}} \cos 4 \theta\right] \\
& \sigma_{22}=p\left[-\frac{a^{2}}{r^{2}}\left(\frac{1}{2} \cos 2 \theta-\cos 4 \theta\right)-\frac{3 a^{4}}{2 r^{4}} \cos 4 \theta\right] \\
& \tau_{12}=p\left[-\frac{a^{2}}{r^{2}}\left(\frac{1}{2} \sin 2 \theta+\sin 4 \theta\right)+\frac{3 a^{4}}{2 r^{4}} \sin 4 \theta\right]
\end{aligned}
$$

To study the accuracy and convergence, the strain energy for several mesh sizes obtained by different methods are plotted in Fig. 11. The parameters here are set to be: Poisson ratio $v=0.3$, Young's modulus $E=90 \mathrm{MPa}$, and $\beta=0.5426$. In the figure, it is found that the strain energy values evaluated from NS-FEM are always greater than the reference exact energy and those obtained by other methods. While the FEM-T3, FEM-Q4, ES-FEM and $\beta$ FEM produce solutions with lower stain energy than the exact one. Again the $\beta$ FEM can provide the super-accurate/close-to-reference results among all these methods. 


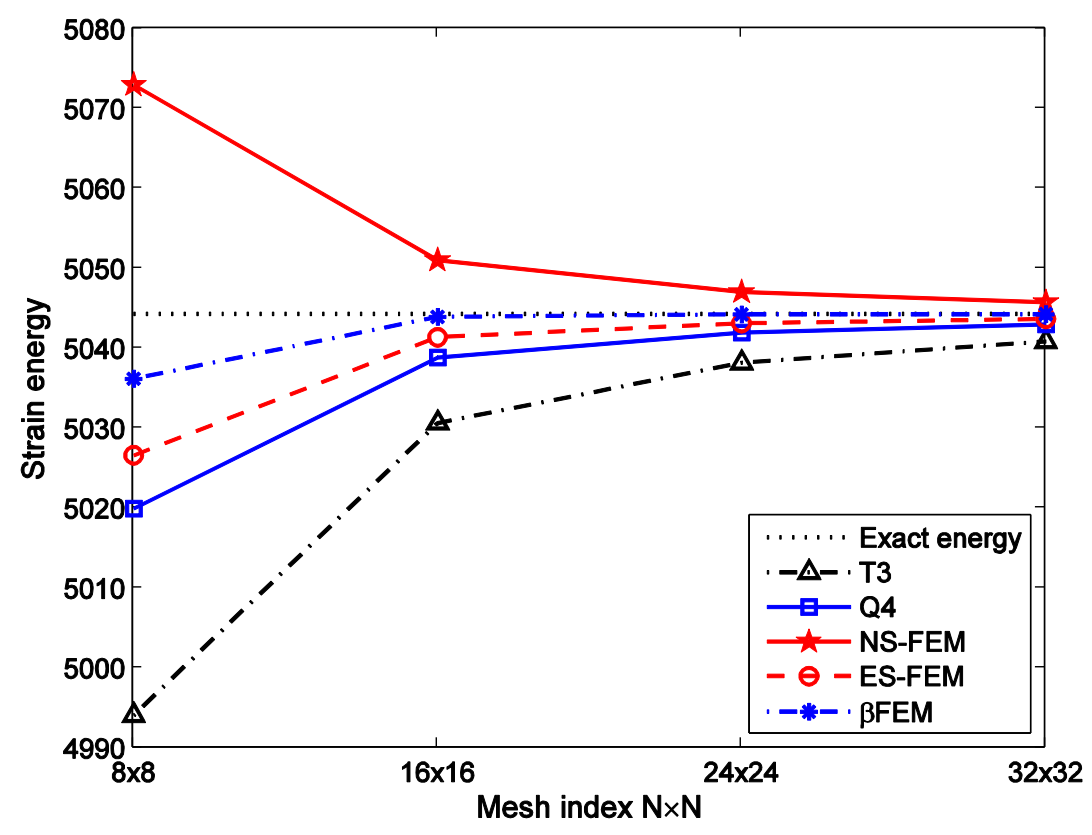

Fig. 11. Solution bounds of energy for a circular hole in an infinite plate under remote tensile load.

In order to check the performance of $\beta \mathrm{FEM}$ in dealing with volumetric locking issue for material with near incompressibility behavior, the incompressibility condition in this example is enforced by setting Poisson's ratio to be close to 0.5 , i.e., $v=0.4 / 0.49 / 0.499 / 0.4999 / 0.49999 / 0.499999 / 0.4999999$. The domain is discretized into triangle mesh with $16 \times 16$ nodes. Table 3 and Fig. 12 show the displacement error norms at different Poisson's ratios for FEM-T3, FEM-Q4, ES-FEM, and $\beta$ FEM with $\beta=1$ (i.e., NS-FEM) and $\beta=0.5+v$. It can be noted that the standard FEM using T3 or Q4 suffers from the volumetric locking. Due to the feature inherited from NS-FEM, which is effective in treating volumetric locking, the $\beta$ FEM using $\beta=1$ or $\beta=0.5+v$ is able to converge to the exact solutions, which indicates its good performance in overcoming volumetric locking for incompressibility.

Table 3 Displacement error norms for infinite plate with a circular hole

\begin{tabular}{|c|c|c|c|c|c|c|}
\hline Mesh & $\begin{array}{l}\text { Poisson's } \\
\text { Ratio }\end{array}$ & FEM-T3 & FEM-Q4 & ES-FEM & $\begin{array}{l}\beta \mathrm{FEM} \\
(\beta=1)\end{array}$ & $\begin{array}{l}\beta \mathrm{FEM} \\
(\beta=0.5+v)\end{array}$ \\
\hline
\end{tabular}




\begin{tabular}{rrrrrrr}
\hline $16 \times 16$ & 0.3 & 0.8283 & 0.2410 & 0.1381 & 0.9499 & 0.5327 \\
$16 \times 16$ & 0.4 & 1.0868 & 0.2985 & 0.1406 & 0.9008 & 0.6398 \\
$16 \times 16$ & 0.49 & 3.9620 & 1.8097 & 0.4339 & 0.8593 & 0.7527 \\
$16 \times 16$ & 0.499 & 7.3455 & 7.6725 & 1.8963 & 0.8557 & 0.7660 \\
$16 \times 16$ & 0.4999 & 8.3564 & 15.1219 & 4.5921 & 0.8556 & 0.8171 \\
$16 \times 16$ & 0.49999 & 8.4904 & 17.4564 & 7.3718 & 0.8555 & 0.7677 \\
$16 \times 16$ & 0.499999 & 8.5044 & 17.7554 & 8.2340 & 0.8555 & 0.7677 \\
$16 \times 16$ & 0.4999999 & 8.5058 & 17.7864 & 8.3464 & 0.8555 & 0.7677 \\
\hline
\end{tabular}

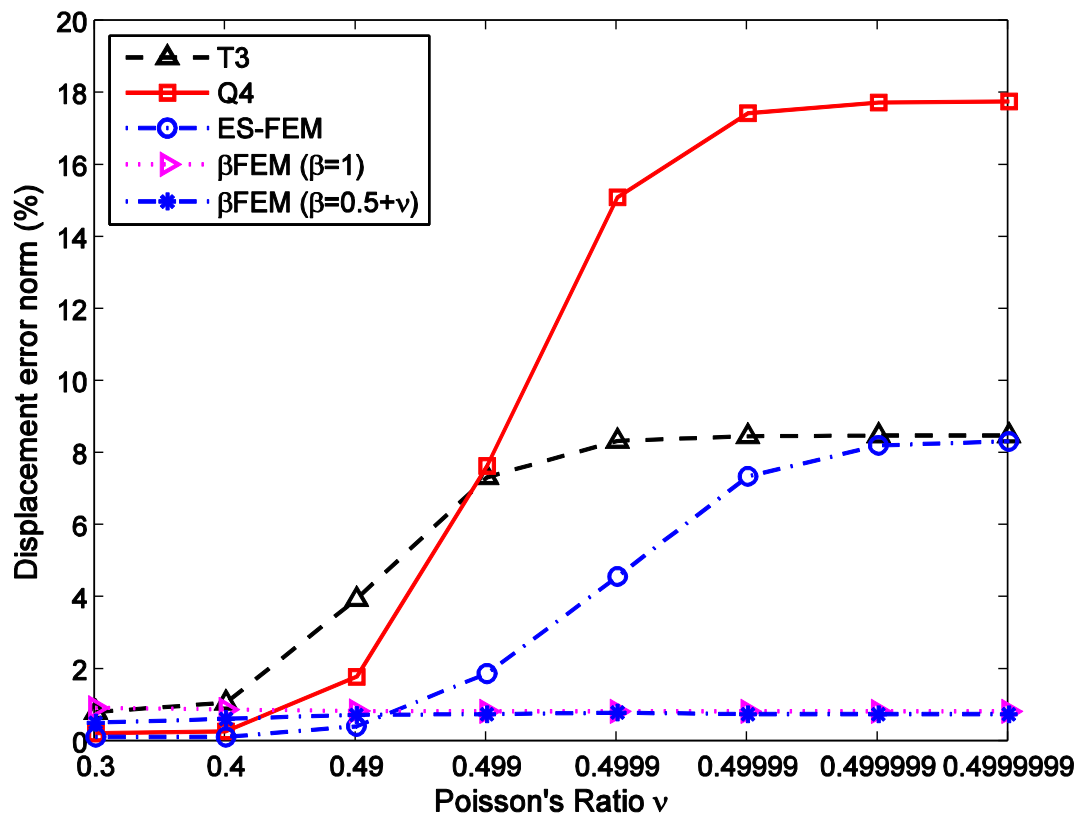

Fig. 12. Displacement error norms of the problem against different Poisson's ratios. 
6.3 Free vibration analysis of an automobile connecting rod: test for temporal stability

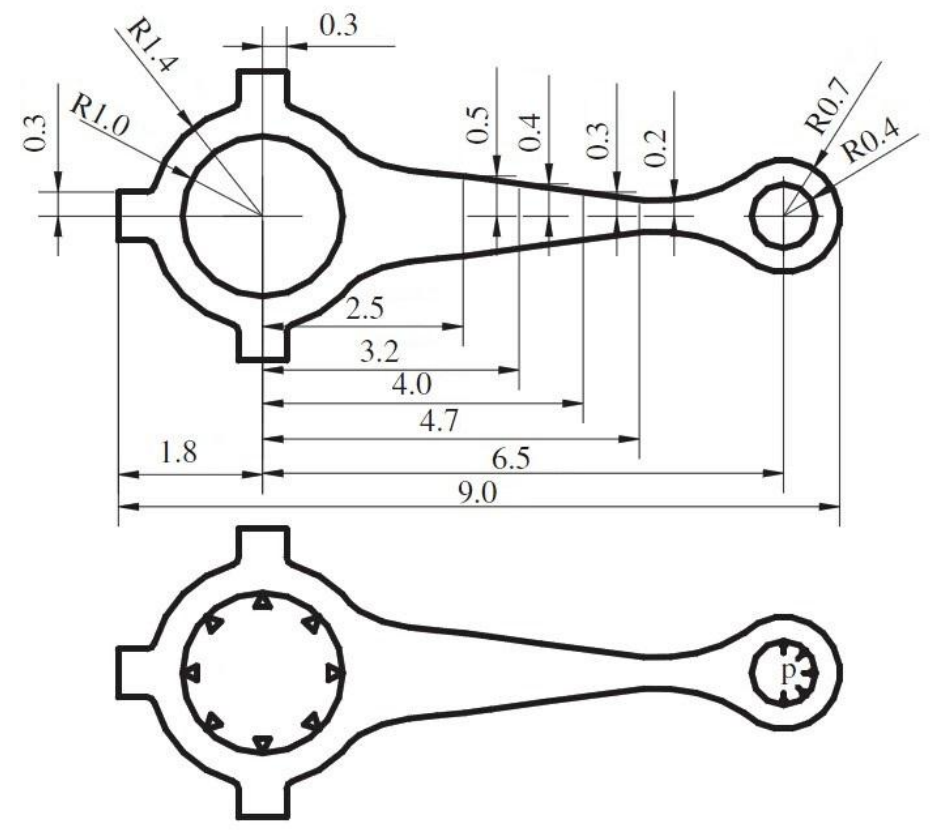

Fig. 13. Geometry and boundary conditions of an automobile connecting rod (from [15]).

As the pure NS-FEM is found to be temporally instable and may have non-zero-energy spurious modes [30], this example will test the temporal stability of $\beta$ FEM by performing a free vibration analysis for an automobile connecting rod. The geometrical dimensions, boundary conditions and loading are illustrated in Fig. 13, with $p=1 \mathrm{MPa}$. The inside circumference for the left side is fixed at both directions. The material parameters are chosen as: Young's modulus $E=3 \times 10^{7} \mathrm{~Pa}$, Poisson's ratio $v=1 / 3$, and mass density $\rho=7.8 \times 10^{3} \mathrm{~kg} / \mathrm{m}^{3}$ for plane stress analysis. The domain is discretized by triangle mesh using 472 nodes for T-mesh based methods (FEM-T3, NS-FEM, ES-FEM and $\beta$ FEM) and 511 nodes for FEM-Q4 as a comparison.

Table 4 lists the first 12 natural frequencies. It is observed that the FEM-T3 has the largest value of frequency and the NS-FEM has the lowest value at each mode, which clearly demonstrates the overly-stiff behavior of FEM-T3 and overly-soft feature of NS-FEM for frequency analysis. All the natural frequencies solved by $\beta$ FEM ( $\beta=0.4$ and 
$\beta=0.6$ ) are bounded between the results from ES-FEM (upper) and NS-FEM (lower).

The mode shapes obtained by NS-FEM and $\beta$ FEM $(\beta=0.6)$ are presented in Fig. 14 and

Fig. 15. It is apparent that the spurious non-zero modes (e.g., modes 5, 9, 11 and 12) exhibited in NS-FEM are vanished in ES-FEM. This example confirms that the $\beta$ FEM with a proper parameter $\beta$ can approach the exact solutions and effectively eliminate the temporal instability and spurious modes, which may have existed in NS-FEM.

Table 4 First twelve natural frequencies $(\mathrm{Hz})$ for the automobile connecting rod

\begin{tabular}{lrrrrrr}
\hline \multirow{2}{*}{ Mesh } & \multicolumn{2}{c}{472 nodes and 736 T3 elements } & & $\begin{array}{r}511 \text { nodes } \\
\text { and 404 Q4 }\end{array}$ \\
\hline \multirow{2}{*}{ Method } & FEM-T3 & NS-FEM & ES-FEM & $\begin{array}{r}\beta \text { FEM } \\
(\beta=0.4)\end{array}$ & $\begin{array}{r}\beta \text { FEM } \\
(\beta=0.6)\end{array}$ & $\begin{array}{r}\text { FEM-Q4 } \\
\text { (Abaqus) }\end{array}$ \\
& 470.37 & 417.68 & 442.70 & 438.94 & 434.15 & 439.74 \\
& 2130.96 & 1911.17 & 2023.13 & 2007.48 & 1987.24 & 2017.5 \\
& 4907.22 & 4683.20 & 4864.00 & 4853.87 & 4839.24 & 4858.8 \\
& 5234.04 & 4746.23 & 4994.71 & 4955.43 & 4903.62 & 5010.4 \\
& 9453.83 & 7742.60 & 8967.82 & 8881.27 & 8763.59 & 9022.2 \\
& 11889.09 & 8167.72 & 11293.10 & 11158.31 & 10963.85 & 11220 \\
& 13906.91 & 9841.74 & 12748.38 & 12530.34 & 12231.46 & 12747 \\
& 16337.07 & 10800.20 & 15426.02 & 15279.60 & 15075.44 & 15240 \\
& 16728.28 & 13271.05 & 15524.57 & 15329.29 & 15095.27 & 15412 \\
& 19935.52 & 14056.13 & 19319.98 & 19179.18 & 18980.37 & 18628 \\
& 20387.98 & 14358.80 & 19712.42 & 19561.28 & 19346.70 & 19571 \\
& 20706.67 & 15890.19 & 19926.97 & 19761.89 & 19533.44 & 19918 \\
\hline
\end{tabular}



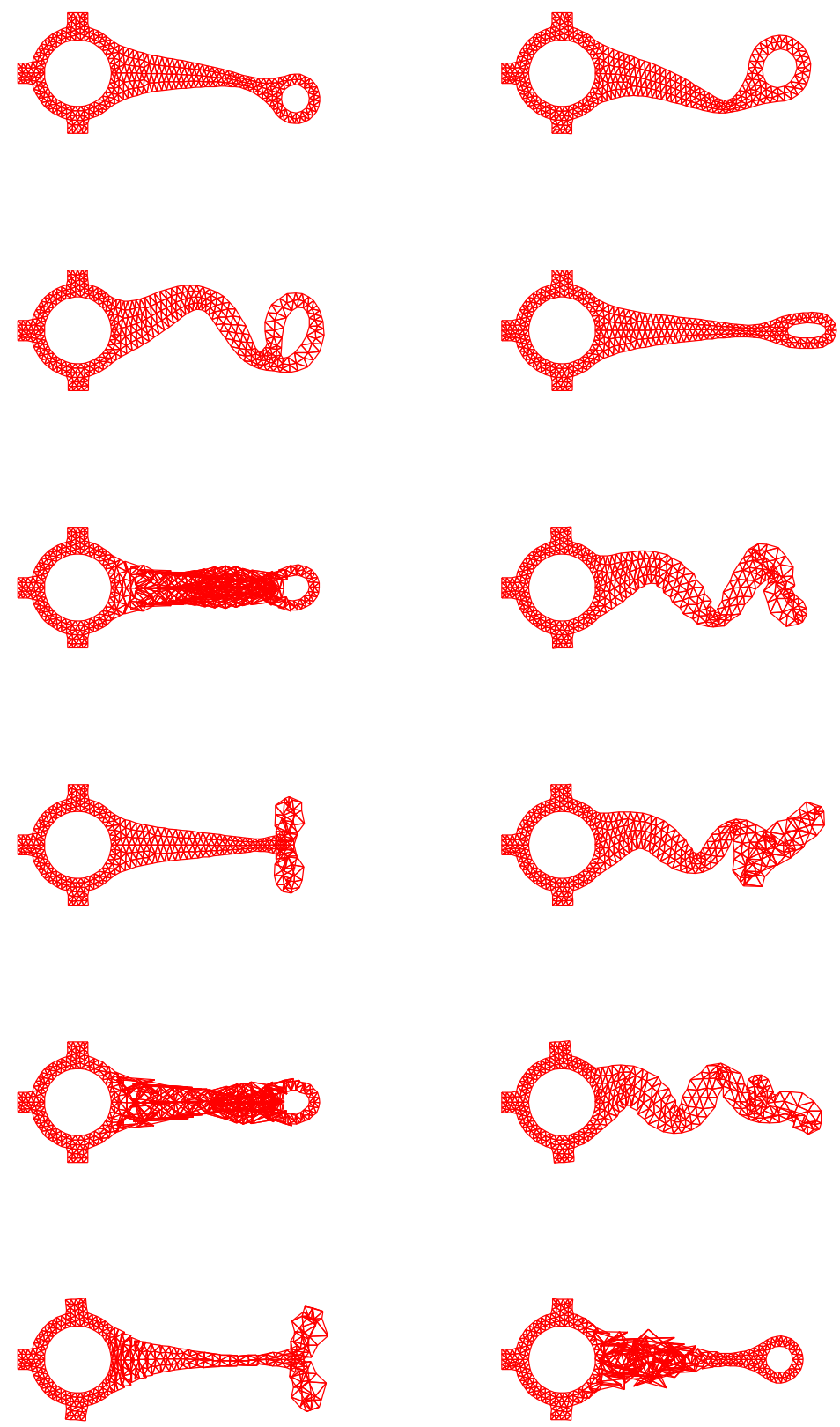

Fig. 14. First 12 modes of the connecting rod obtained by NS-FEM. 

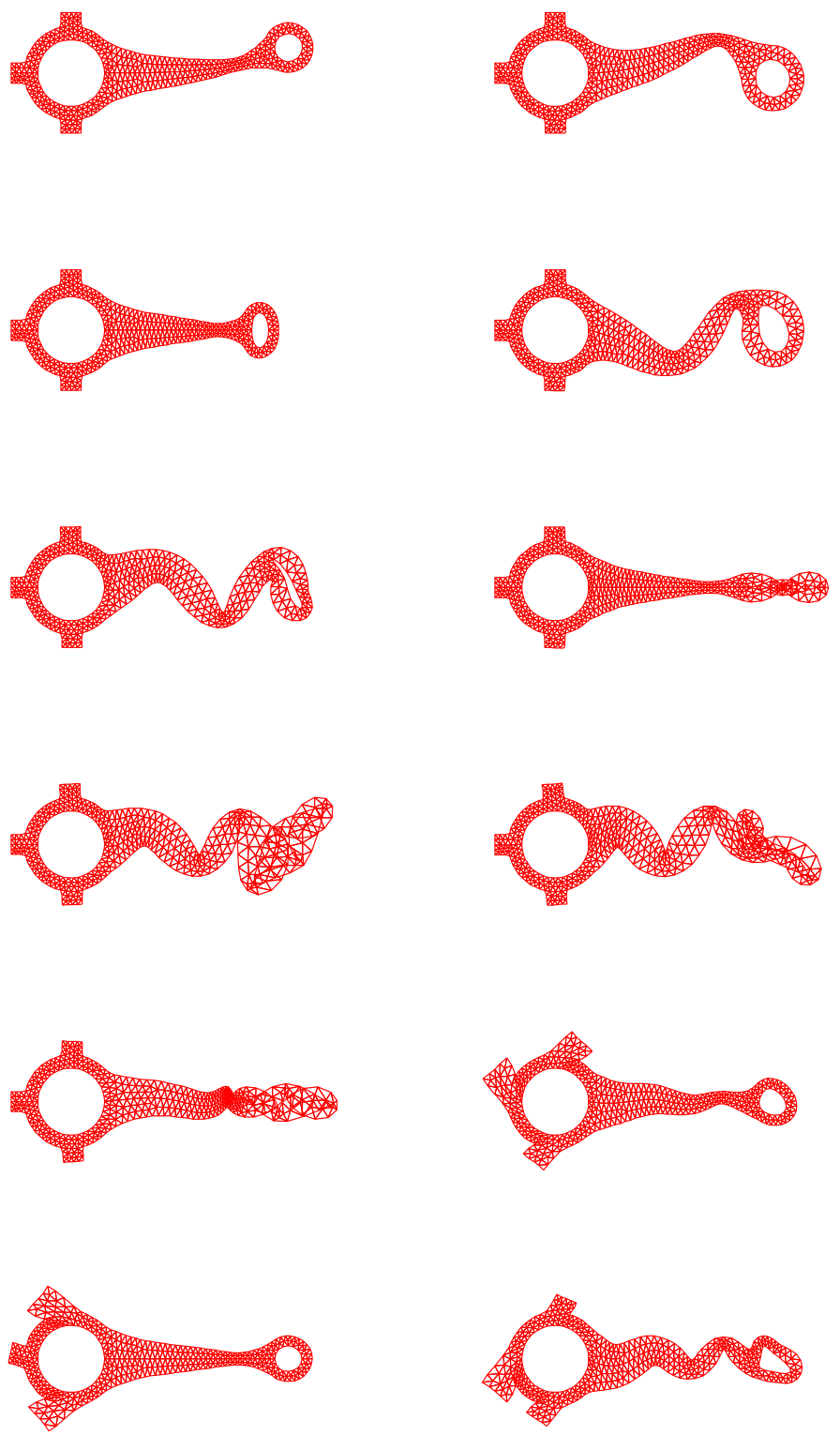

Fig. 15. First 12 modes of the connecting rod obtained by $\beta$ FEM. 


\subsection{A 3D cantilever of cubic shape: accuracy study}

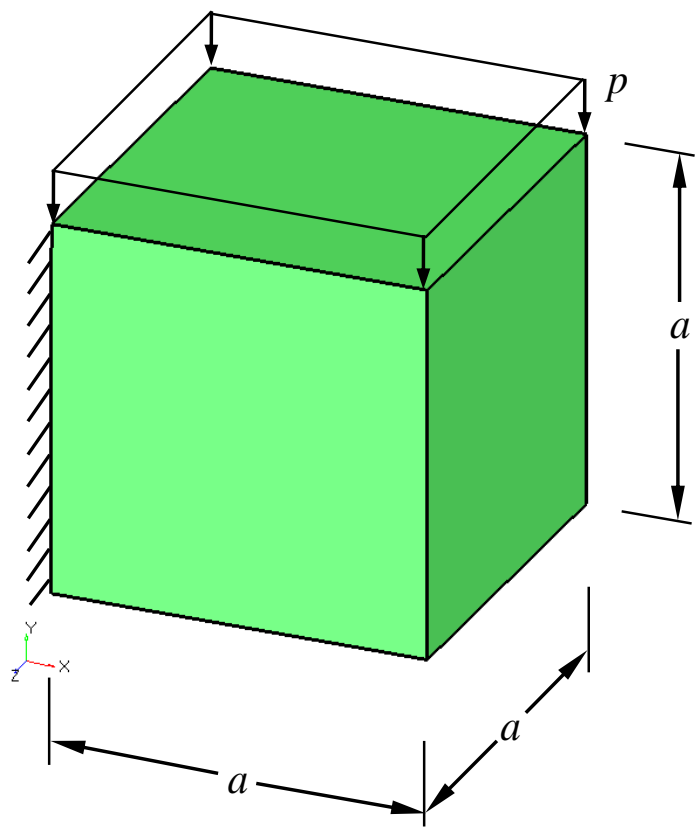

(a)

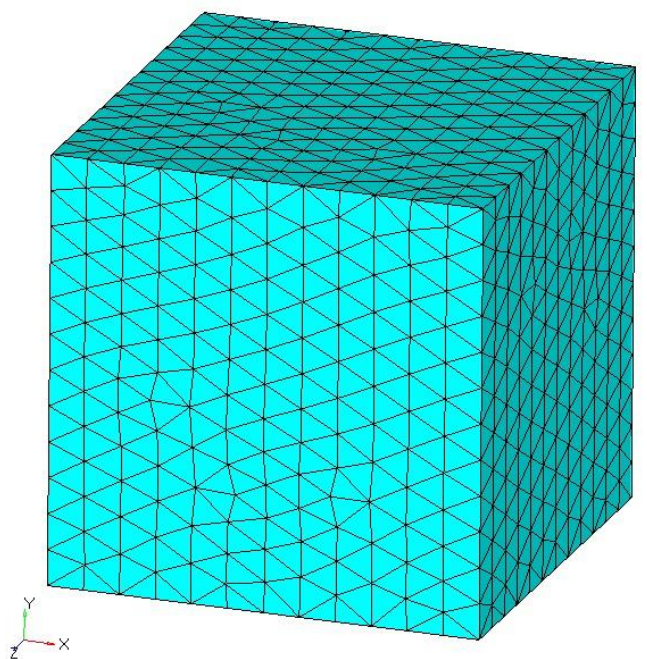

(b)

Fig. 16. A cubic cantilever submitted to a uniform pressure on its upper face: (a) geometry and (b) domain discretization by tetrahedral mesh.

This example considers a 3D cantilever with cubic shape as shown in Fig. 16(a), which is submitted to a uniform pressure on its top face. The input parameters are set as: $a=1.0, p=1.0, E=1.0$ and $v=0.25$. The exact solution of strain energy is unknown for this problem, but a reference solution is available in [46], which applied Richardson's extrapolation [47] on the solutions of hexahedral super-elements. The approximation of strain energy reported in this reference is 0.950930 . Another reference solution of strain energy is 0.9486 [25], which was obtained from FEM model with very fine mesh using second-order 10-node tetrahedral elements (T-10). In $\beta$ FEM computations, three types of mesh structures (M1: 203 nodes and 611 elements; M2: 554 nodes and 1936 elements; and M3: 1418 nodes and 5554 elements, shown in Fig. 16(b)) are adopted. The estimated values of energy from different values of $\beta$ are plotted and compared in 
Fig. 17. In order to approximate good results, the parameter $\beta \in[0.8,0.9]$ is suggested to adopt for this problem. For mesh structure M2 and M3, the close-to-reference solution can be obtained at $\beta \cong 0.84$.

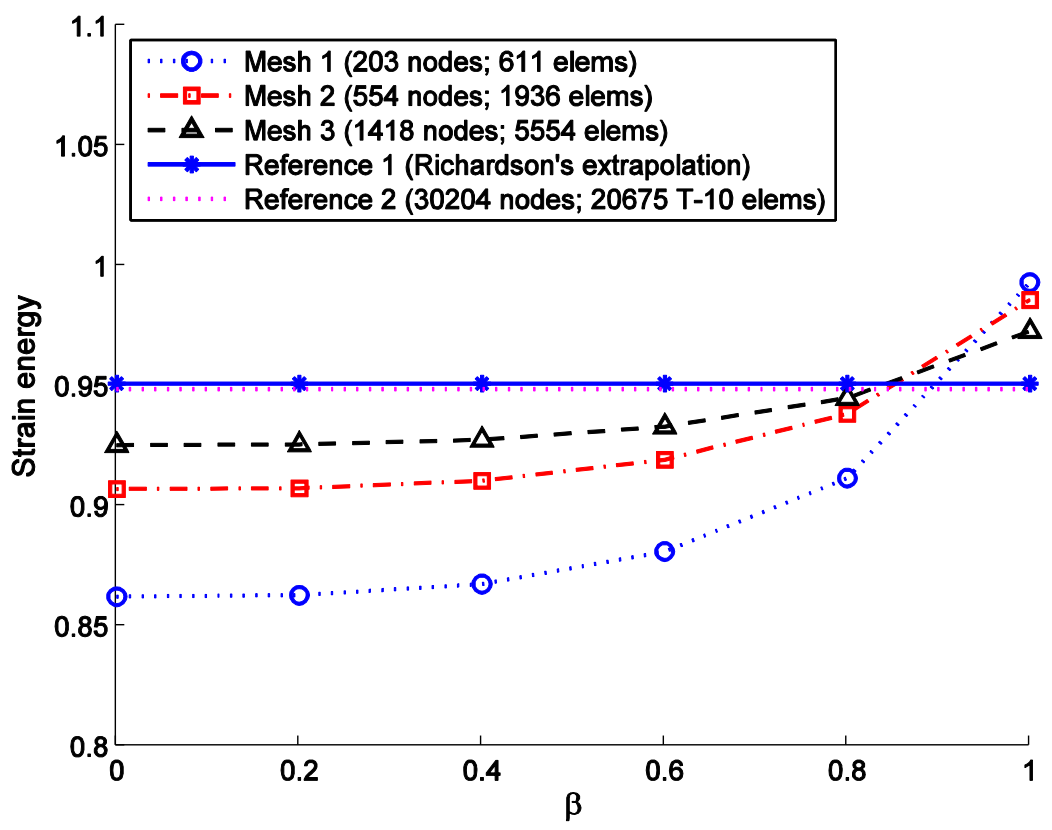

Fig. 17. Strain energy of cubic cantilever obtained by $\beta$ FEM. 


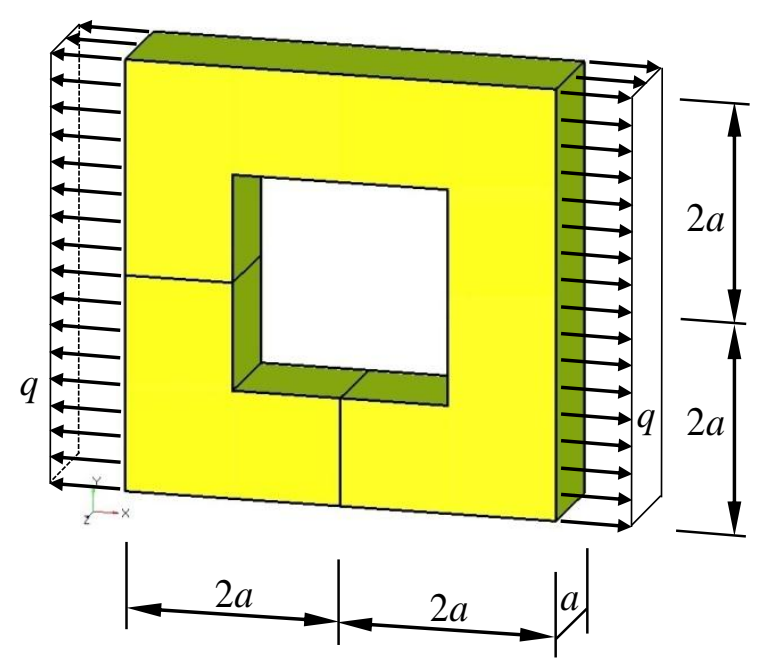

(a)

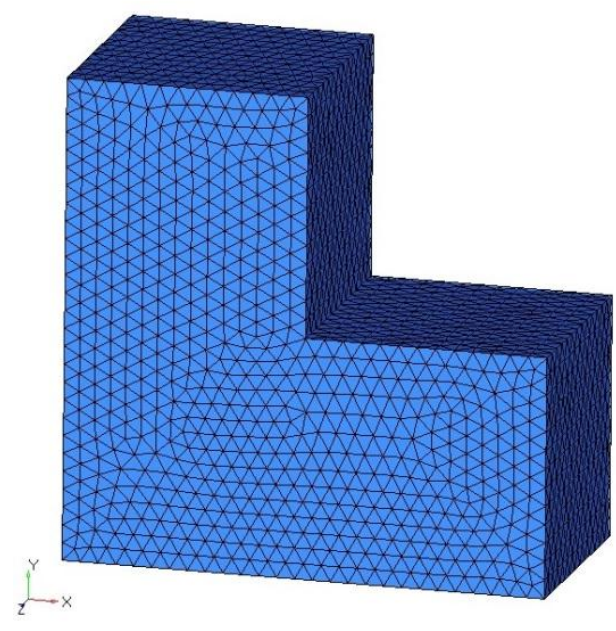

(b)

Fig. 18. A 3D L-shaped problem and its quarter model: (a) geometry and (b) a quarter model discretized by tetrahedral mesh.

Now consider a 3D square block with a rectangular parallelepiped hole in Fig. 18(a), which is subjected to a uniform traction $q$ on left and right side faces. Since the problem is biaxial symmetry, only a quadrant of the block (L-shaped) needs to be modeled as shown in Fig. 18(b). The parameters are assumed to be: $a=1.0, q=1.0$, $E=1.0$ and $v=0.3$. Cugnon [48] provided an approximation of the reference strain energy to be 6.19985060 . The results of strain energy computed from $\beta$ FEM are plotted in Fig. 19, which indicate that the parameter $\beta \cong 0.8$ is recommended to get the close-to-reference solutions. 


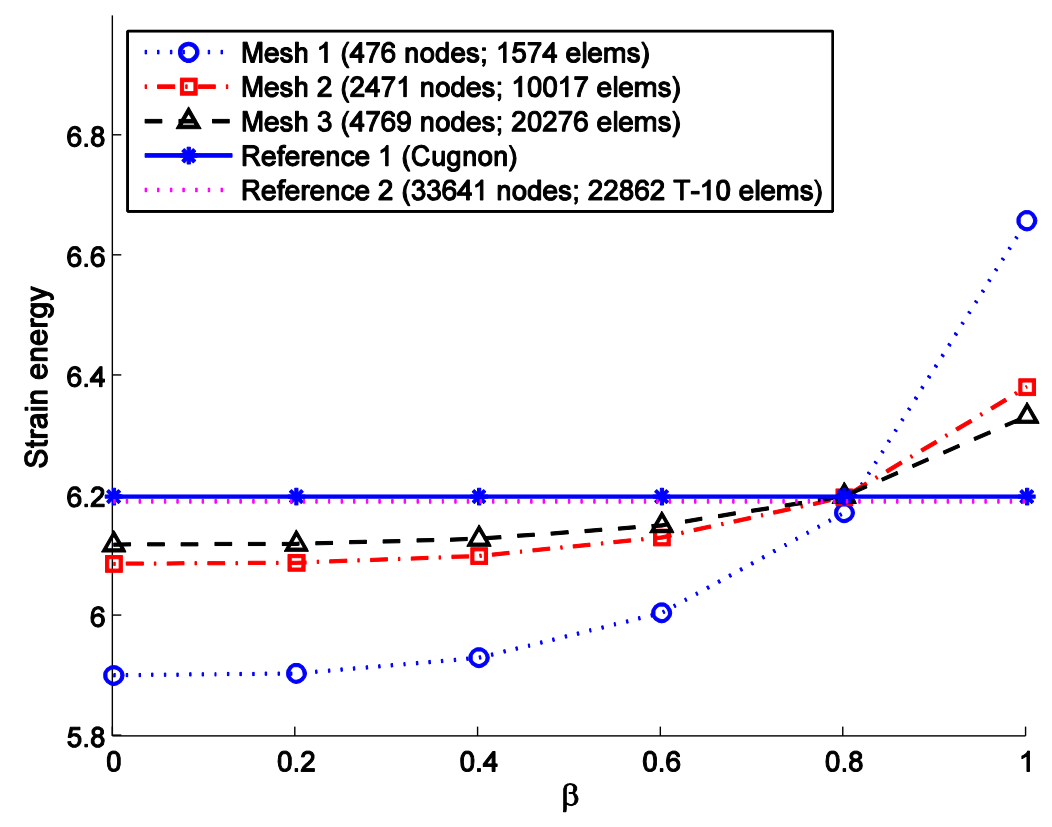

Fig. 19. Strain energy of the 3D L-shaped problem obtained by $\beta$ FEM.

\subsection{A molar tooth: analysis for problem with complex geometry}

In this example, a human mandibular molar tooth under vertical pressure will be simulated. The aim of the present study is to show the advantage of $\beta$ FEM in modeling object with moderate complex shape of geometry, since the adopted element type (tetrahedron) will be the only option to discretize arbitrary irregular complex shapes. Fixed zero-displacement in the three spatial dimensions are assigned to the nodes below the horizontal plane at $z=0.6$. As the data of true loading of mastication may be a stochastic event and will be difficult to determine, here we assume a uniformly vertical downward loading applied at the surface above the horizontal plane $z=10.2$ (above the lowest point of top surface). The value of total force using for analysis is set to be $225 \mathrm{~N}$ [49]. The material is assumed to be a homogeneous and isotropic material which has the same properties as tooth enamel: $E=84.1 \mathrm{GPa}[50]$ and $v=0.30 \quad$ [51]. 

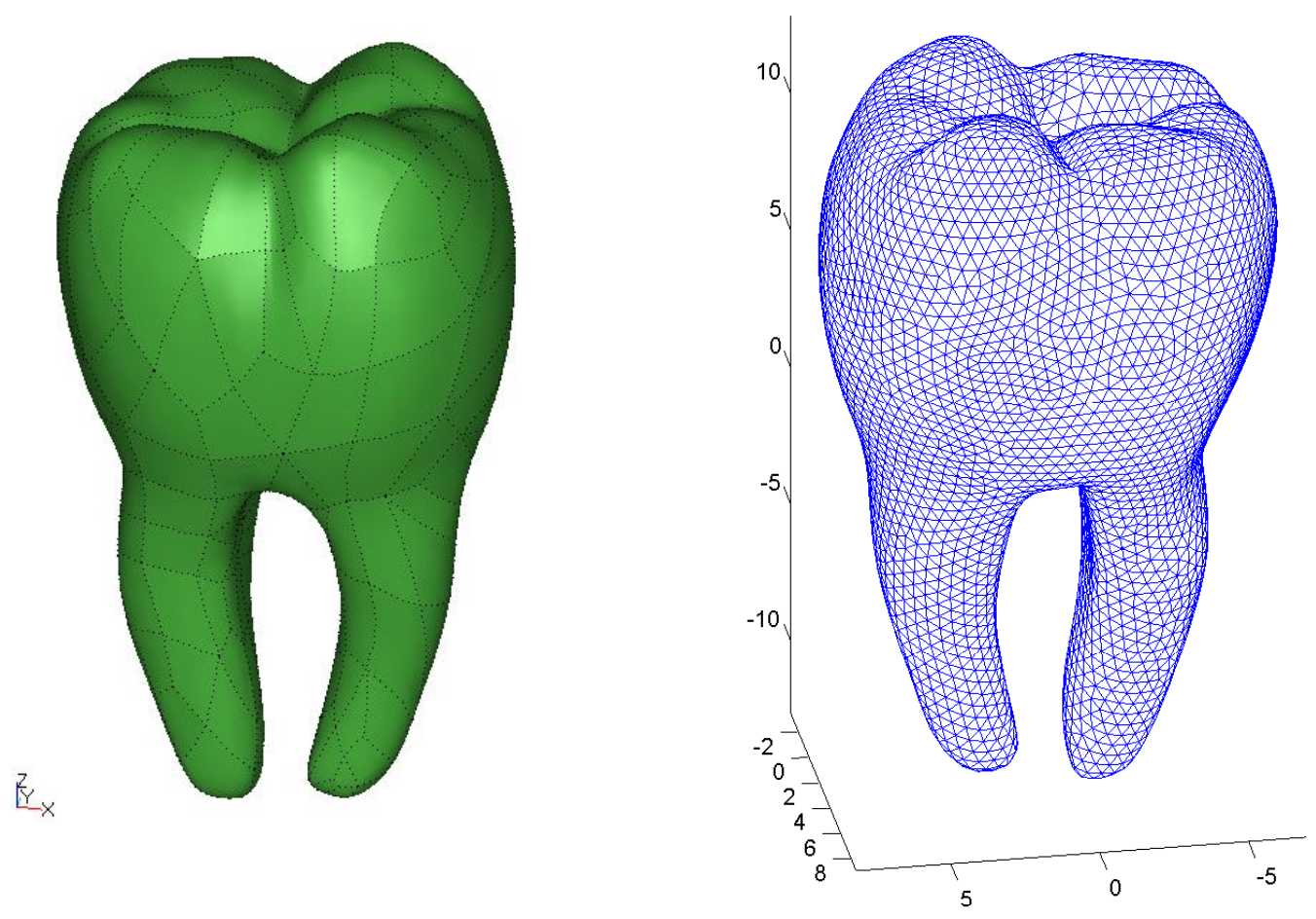

(a)

(b)

Fig. 20. A human molar tooth and its representative mesh: (a) geometry and (b) a model discretized by T-mesh using 33968 tetrahedral elements.

The stress distribution obtained from presented $\beta$ FEM is displayed in Fig. 21 and it is compared with the FEM results obtained from ABAQUS, which utilizes the same mesh structure and loading/boundary conditions. It is noticed that the input data of this problem is simplified and some assumptions are made as the related experimental data is unknown. However, it is already demonstrated the potential of the present method for investigating the stress distribution of dental structures. Also it can be used in the field of creating patient-specific models for clinical operations of tooth restoration and dental implants. The study here shows the capability of our $\beta$ FEM in modeling complex 3D geometry for 
medical applications (i.e., dental) and simulations of biological systems with irregular shapes.

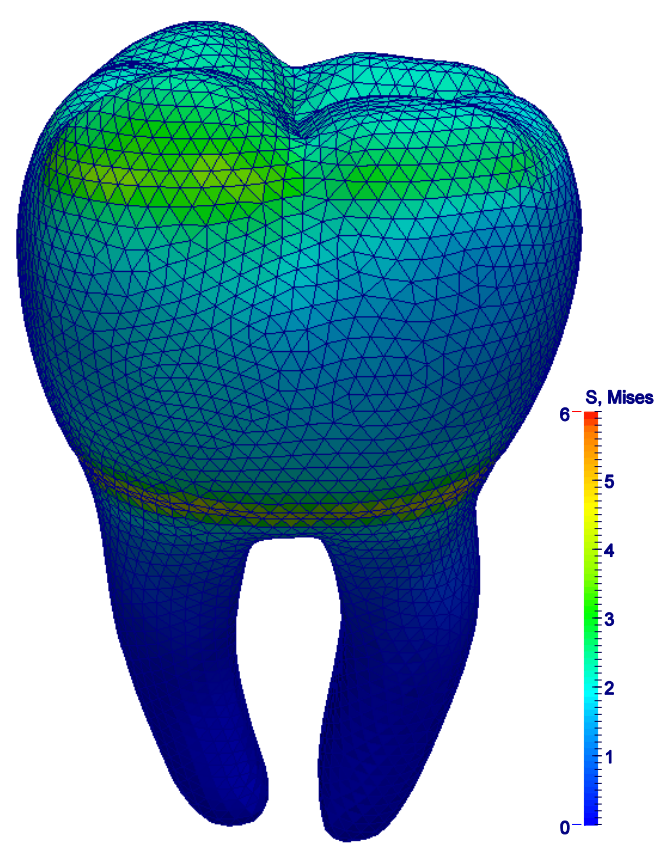

(a)

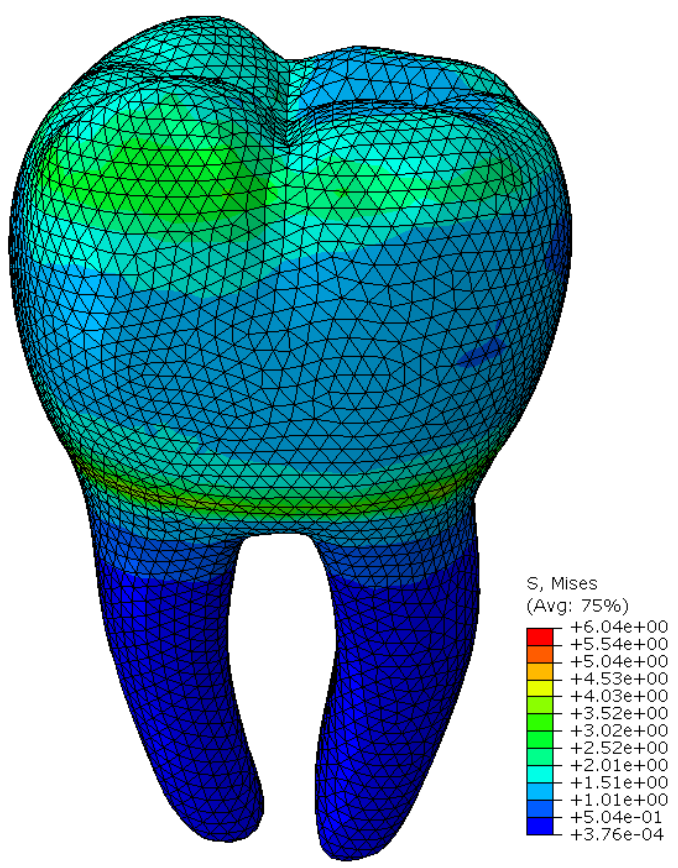

(b)

Fig. 21. Stress distribution of molar tooth analysis using T-mesh: (a) $\beta$ FEM model and (b) FEM model by ABAQUS.

\subsection{A 3D cantilever beam subjected to a regular distributed load: analysis for large deformation problem}

A large deformation analysis of a $3 \mathrm{D}$ cantilever beam is performed using $\beta \mathrm{FEM}$ in this example. The geometrical dimensions of the beam are given as: $2 \mathrm{~cm} \times 2 \mathrm{~cm} \times 10 \mathrm{~cm}$. The beam is constrained at its left end and subjected to a regular distributed loading at its right end. The material parameters are set as $E=30 \mathrm{GPa}$ and $v=0.30$. The domain is discretized by a mesh using 117 nodes and 298 elements. The geometrically nonlinear analysis is based on total Lagrange formulation using 10 increment steps 
( nsteps $=10$ ) with $\Delta f=2 \mathrm{kN} / \mathrm{cm}^{2}$ in each step. In Fig. 22, the initial grid and final deformed configuration for the model are shown. The tip deflections / vertical displacement $(\mathrm{cm})$ at each load step obtained by different methods are compared in Table 5 and Fig. 23. It is apparent in each step, the problem converges quickly as all the iterations at each step are no more than 5. Compared to linear problem, the nonlinear large deformation analysis models behaves stiffer and unpliable to bend. The deflections obtained by $\beta$ FEM ( $\beta=0.8985)$ are softer than those from FEM-T4 and FS-FEM, and they are closer to the model using 8-node hexahedral elements (FEM-H8) [24] with fine mesh.
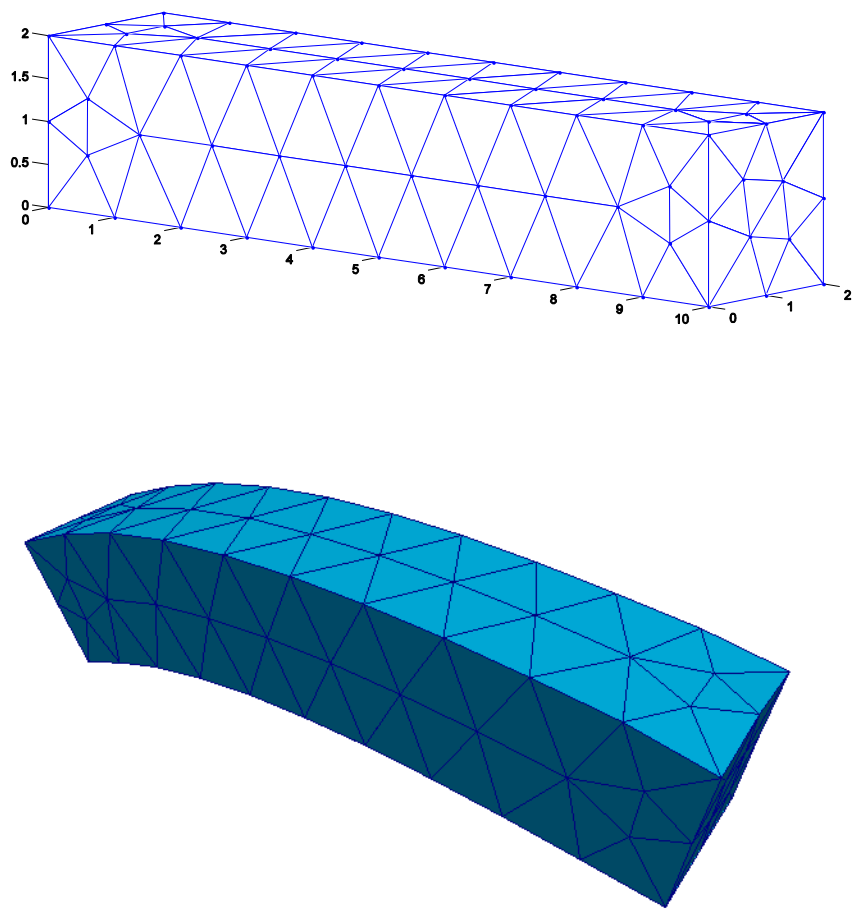

Fig. 22. Initial mesh and final deformed configuration of the 3D cantilever beam problem obtained by $\beta$ FEM based on geometrically nonlinear analysis.

Table 5 Tip deflections $(\mathrm{cm})$ at each load step for 3D cantilever beam. 


\begin{tabular}{lccccc}
\hline Load step (n) & $\begin{array}{c}\text { FEM-T4 } \\
\text { (linear, 1322 } \\
\text { nodes) }\end{array}$ & $\begin{array}{c}\text { FEM-T4 } \\
\text { (nonlinear, } \\
117 \text { nodes) }\end{array}$ & $\begin{array}{c}\text { FS-FEM } \\
\text { (nonlinear, } \\
117 \text { nodes) }\end{array}$ & $\begin{array}{c}\text { FEM-H8 } \\
\text { (nonlinear, } \\
1323 \text { nodes) }\end{array}$ & $\begin{array}{c}\beta \text { FEM } \\
\text { (nonlinear, } \\
117 \text { nodes) }\end{array}$ \\
\hline 1 & 0.2364 & $0.1333(3)^{*}$ & $0.1486(3)$ & $0.2421(3)$ & $0.1969(3)$ \\
2 & 0.4728 & $0.2658(3)$ & $0.2962(2)$ & $0.4522(3)$ & $0.4016(5)$ \\
3 & 0.7092 & $0.3988(2)$ & $0.4448(2)$ & $0.6405(3)$ & $0.6047(3)$ \\
4 & 0.9456 & $0.5320(2)$ & $0.5940(2)$ & $0.8205(3)$ & $0.7985(2)$ \\
5 & 1.1819 & $0.6641(2)$ & $0.7419(2)$ & $1.0022(4)$ & $0.9907(2)$ \\
6 & 1.4183 & $0.7949(2)$ & $0.8880(2)$ & $1.1762(4)$ & $1.1840(2)$ \\
7 & 1.6547 & $0.9242(2)$ & $1.0321(2)$ & $1.3495(4)$ & $1.3735(2)$ \\
8 & 1.8911 & $1.0519(2)$ & $1.1741(2)$ & $1.5222(4)$ & $1.5590(2)$ \\
9 & 2.1275 & $1.1779(2)$ & $1.3138(2)$ & $1.6943(4)$ & $1.7390(2)$ \\
10 & 2.3639 & $1.3022(2)$ & $1.4510(2)$ & $1.8656(4)$ & $1.9131(2)$ \\
\hline
\end{tabular}

* The number in the bracket is the iterations in each step.

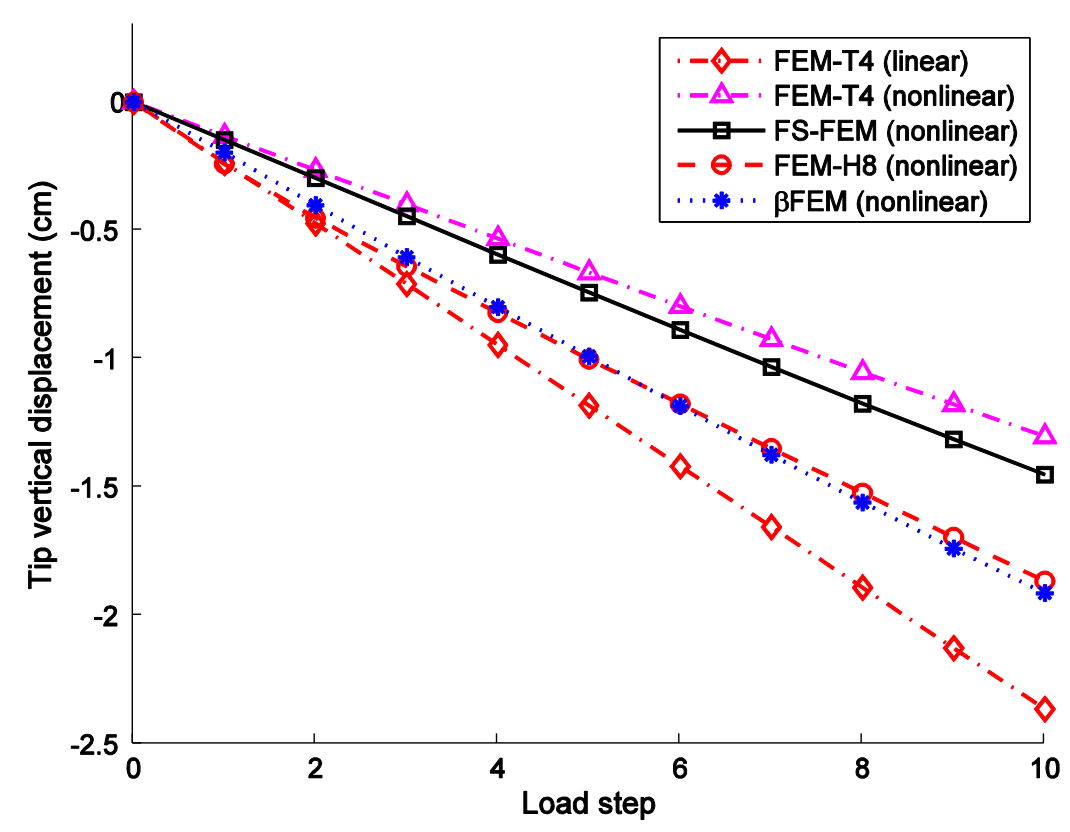

Fig. 23. Tip deflection $(\mathrm{cm})$ at each load step for the 3D cantilever beam problem.

\section{Conclusions}


In this work, a novel smoothed techniques based generalized beta finite element method $(\beta \mathrm{FEM})$ has been developed and applied for both $2 \mathrm{D}$ and 3D solid mechanics problems. For $\beta$ FEM, smoothing domains generated from both edge-based (2D)/face-based (3D) and node-based strain smoothing techniques are employed to construct a smoothed model. A key aspect of the method is that it inherits the features of both the NS-FEM and ES/FS-FEM. Standard patch tests are likewise satisfied. Through the present framework of formulations and numerical discussions by examples, the major characteristics of the proposed method have been demonstrated and verified, including high accuracy, insensitivity to mesh quality or distortion, immunity or alleviation of volumetric locking, temporal stability and capability for modeling objects with complex geometry. From these studies, some conclusions can be drawn as following:

(1) An adjustable parameter $\beta$ controls the portion of area of edge/face-based and node-based smoothing domains. In the context of elasticity, it is promising to find nearly exact solution in strain energy due to solution shifting by tuning the adjustable parameter, since the exact solution will be within the narrow interval bounded by the solutions of $\beta$ FEM with $\beta=0$ and $\beta=1$.

(2) The method can be immune from volumetric locking and no special treatments are required for modeling nearly incompressible materials. This feature is inherited from NS-FEM by properly choosing the parameter $\beta$ of $\beta$ FEM, and the ES/FS-FEM may not possess this property due to its inherent defect.

(3) Temporal instability and spurious modes existed in NS-FEM can be eliminated by the proposed $\beta$ FEM model with a proper parameter $\beta$. However, the effective way to find such a parameter for different problems needs to be further studied.

(4) The method is effective in analysis of solid mechanics problems for both linear and geometrically nonlinear cases. 
(5) Due to the fact that tetrahedral grids can be efficiently generated for complex geometry using fully automatic procedures, the method has a lot of potential and capabilities for application in complex-geometry areas (such as biomedical, automotive, and aerospace engineering) as the algorithms for $\beta$ FEM are based upon T-mesh.

\section{Acknowledgments}

The senior author would like to thank the support partially from: (1) US NSF Grant under the award No. DMS-1214188; (2) Open Research Funding Program of the State Key Laboratory of Advanced Design and Manufacturing for Vehicle Body (DMVB), Hunan University, under the grant No. 41215002.

\section{References}

[1] Liu GR, Quek SS. The Finite Element Method: A Practical Course (2nd edn). Butterworth-Heinemann: Oxford, 2013.

[2] Onishi Y, Amaya K. A locking-free selective smoothed finite element method using tetrahedral and triangular elements with adaptive mesh rezoning for large deformation problems. Int J for Numer Meth Eng 2014; 99(5):354-71.

[3] Allman DJ. A compatible triangular element including vertex rotations for plane elasticity analysis. Comput Struct 1984; 19(2):1-8.

[4] Allman DJ. Evaluation of the constant strain triangle with drilling rotations. Int $\mathbf{J}$ for Numer Meth Eng 1988; 26(12):2645-55. 
[5] Chen HC. Evaluation of Allman triangular membrane element used in general shell analyses. Comput Struct 1992; 43(5):881-7.

[6] Huang M, Zhao Z, Shen C. An effective planar triangular element with drilling rotation. Finite Elem Anal Des 2010; 46:1031-6.

[7] Piltner R., Taylor RL. Triangular finite elements with rotational degrees of freedom and enhanced strain modes. Comput Struct 2000; 75:361-8.

[8] Bergan PG, Felippa CA, A triangular membrane element with rotational degrees of freedom. Comput Methods Appl Mech Engrg 1985; 50:25-69.

[9] Cook RD. Modified formulations for nine-dof plane triangles that include vertex rotations. Int J for Numer Meth Eng 1991; 31:825-35.

[10] Dohrmann CR, Key SW, Heinstein MW, Jung J. A least squares approach for uniform strain triangular and tetrahedral finite elements. Int $\mathbf{J}$ for Numer Meth Eng $1998 ; 42: 1181-97$.

[11] Dohrmann CR, Heinstein MW, Jung J, Key SW, Witkowski WR. Node-based uniform strain elements for three-node triangular and four-node tetrahedral meshes. Int J for Numer Meth Eng 2000; 47:1549-68.

[12] Hong WI, Kim YH, Lee SW. An assumed strain triangular solid shell element with bubble function displacements for analysis of plates and shells. Int $\mathrm{J}$ for Numer Meth Eng 2001; 52(4):455-69

[13] Liu GR, Nguyen-Thoi T, Dai KY, Lam KY. Theoretical aspects of the smoothed finite element method (SFEM). Int J for Numer Meth Eng 2007; 71(8):902-30.

[14] Nguyen-Thoi T, Liu GR, Dai KY, Lam KY. Selective smoothed finite element method. Tsinghua Sci Technol 2007; 12(5):497-508.

[15] Liu GR, Nguyen-Thoi T, Lam KY. An edge-based smoothed finite element method (ES-FEM) for static, free and forced vibration analyses in solids. J Sound Vib 2009; 320:1100-30. 
[16] Liu GR, Dai KY, Nguyen-Thoi T. A smoothed finite element for mechanics problems. Finite Elem Anal Des 2007; 39(6):859-77.

[17] Zeng W, Liu GR, Kitamura Y, Nguyen-Xuan H. A three-dimensional ES-FEM for fracture mechanics problems in elastic solids. Eng Fract Mech 2013; 114:127-50.

[18] Liu GR, Nguyen-Thoi T, Lam KY. A node-based smoothed finite element method for upper bound solution to solid problems (NS-FEM). Comput Struct 2009; 87:1426.

[19] Zeng W, Liu GR, Jiang C, Dong XW, Chen HD, Bao Y, Jiang Y. An effective fracture analysis method based on the virtual crack closure-integral technique implemented in CS-FEM. Appl Math Model 2016; 40: 3783-800.

[20] Tang LM, Chen WJ, Liu XY. String net function approximation and quasi-conforming technique. In Hybrid and Mixed Finite Element Methods, Chapter 9, Atluri SA et al. (eds). Wiley: New York, 1983.

[21] Chen JS, Wu CT, Yoon S, You Y. A stabilized conforming nodal integration for Galerkin mesh-free methods. Int J for Numer Meth Eng 2001; 50(2):435-66.

[22] Liu MB, Liu GR (2010) Smoothed particle hydrodynamics (SPH): an overview and recent developments. Arch Comput Methods Eng 17:25-76.

[23] Yoo JW, Moran B, Chen J-S. Stabilized conforming nodal integration in the natural-element method. Int J for Numer Meth Eng 2004; 60(5):861-90.

[24] Liu GR, Nguyen-Thoi T. Smoothed Finite Element Methods. CRC Press, Taylor and Francis Group: New York, 2010.

[25] Nguyen-Thoi T, Liu GR, Lam KY. A face-based smoothed finite element method (FS-FEM) for 3D linear and nonlinear solid mechanics problems using 4-node tetrahedral elements. Int J for Numer Meth Eng 2009; 78:324-53.

[26] Dai KY, Liu GR. Free and forced vibration analysis using the smoothed finite element method (SFEM). J Sound Vib 2007; 301(3-5):803-20. 
[27] Zeng W, Larsen JM, Liu GR. Smoothing technique based crystal plasticity finite element modeling of crystalline materials. Int J Plasticity 2015; 65:250-68.

[28] Chen L, Liu GR, Nourbakhsh-Nia N, Zeng K. A singular edge-based smoothed finite element method (ES-FEM) for bimaterial interface cracks. Comput Mech 2010; 45(2-3):109-25.

[29] Jiang C, Liu GR, Han X, Zhang Z-Q, Zeng W. A smoothed finite element method for analysis of anisotropic large deformation of passive rabbit ventricles in diastole. Int J Numer Meth Biomed Engng 2015; 31(1):e02697.

[30] Jiang C, Zhang Z-Q, Liu GR, Han X, Zeng W. An edge-based/node-based selective smoothed finite element method using tetrahedrons for cardiovascular tissues. Eng Anal Bound Elem 2015; 59:62-77.

[31] Nguyen-Thoi T, Liu GR, Nguyen-Xuan H, Additional properties of the node-based smoothed finite element method (NS-FEM) for solid mechanics problems. Int $\mathbf{J}$ Comput Meth 2009; 6(4):633-66.

[32] Liu GR, Nguyen-Xuan H, Nguyen-Thoi T. A theoretical study on the smoothed FEM (S-FEM) models: Properties, accuracy and convergence rates. Int $\mathrm{J}$ for Numer Meth Eng 2010; 84(10):1222-56.

[33] Liu GR. A G space theory and a weakened weak (W2) form for a unified formulation of compatible and incompatible methods: Part I theory. Int J for Numer Meth Eng 2010; 81:1093-126.

[34] Zeng W, Liu GR, Li D, Dong XW. A smoothing technique based beta finite element method ( $\beta$ FEM) for crystal plasticity modeling. Comput Struct 2016; 162:48-67.

[35] Zhang ZQ, Liu GR. An edge-based smoothed finite element method (ES-FEM) using 3-node triangular elements for 3D non-linear analysis of spatial membrane structures. Int J for Numer Meth Eng 2011; 86(2): 135-54. 
[36] Nguyen-Thoi T, Liu GR, Vu-Do HC, Nguyen-Xuan H. A face-based smoothed finite element method (FS-FEM) for visco-elastoplastic analyses of 3D solids using tetrahedral mesh. Comput Methods Appl Mech Engrg 2009; 198(41-44): 3479-98.

[37] Liu GR, Nguyen-Thoi T, Lam KY. A novel alpha finite element method ( $\alpha$ FEM) for exact solution to mechanics problems using triangular and tetrahedral elements. Comput Methods Appl Mech Engrg 2008; 197 (45-48):3883-97.

[38] Bathe KJ. Finite Element Procedures (2nd edn). Klaus-Jürgen Bathe: Watertown, MA, 2014.

[39] De Borst R, Crisfield MA, Remmers JJC, Verhoosel CV. Nonlinear Finite Element Analysis of Solids and Structures (2nd edn). Wiley: West Sussex, UK, 2012.

[40] Reddy JN. An Introduction to Nonlinear Finite Element Analysis. Oxford University Press: Oxford, 2004.

[41] Wu F, Liu GR, Li GY, He ZC. A new hybrid smoothed FEM for static and free vibration analyses of Reissner-Mindlin Plates. Comput Mech 2014: 1-26.

[42] Cook RD. Improved two-dimensional finite element. Journal of the Structural Division 1974; ASCE 100 (ST6):1851-63.

[43] Taylor RL, Beresford PJ, Wilson EL. A non-conforming element for stress analysis. Int J for Numer Meth Eng 1976; 10:1211-19.

[44] Fredriksson M, Ottosen NS. Fast and accurate four-node quadrilateral. Int J for Numer Meth Eng 2004; 61:1809-34.

[45] Timoshenko SP. Goodier JN. Theory of elasticity (3rd edn)., McGraw-Hill: New York, 1970.

[46] Pereira OJBA. Hybrid equilibrium hexahedral elements and super-elements. Comm Numer Meth Eng 2008; 24(2):157-65.

[47] Richardson LF. The approximate arithmetical solution by finite differences of physical problems involving differential equations, with an application to the stresses in a masonry dam. Philos T Roy Soc A 1910; 210:307-57. 
[48] Cugnon F. Automatisation des calculs elements finis dans le cadre de la methode-p, Universite de Lie, Ph.D. Thesis, 2000.

[49] Magne P. Efficient 3D finite element analysis of dental restorative procedures using micro-CT data. Dent Mater 2007; 23(5):539-48.

[50] Craig RG, Peyton FA, Johnson DW. Compressive properties of enamel, dental cements, and gold. J Dent Res 1961; 40:936-45.

[51] Anusavice KJ, Hojjatie B. Influence of incisal length of ceramic and loading orientation on stress distribution in ceramic crowns. J Dent Res 1988; 67:1371-5. 